\title{
Saisonnalité du transport de carbone organique dissous dans le ruisseau de l'Hermine, un bassin versant de tête de réseau du Bouclier Canadien
}

\author{
Seasonal variations of the transport of dissolved organic \\ carbon in the intermittent stream draining the Hermine \\ headwater catchment on the Canadian Shield
}

\author{
M.-C. Turmel, J. M.L. Turgeon, C. François et B. Cloutier-Hurteau
}

Volume 18, numéro 3, 2005

URI : https://id.erudit.org/iderudit/705563ar

DOI : https://doi.org/10.7202/705563ar

\section{Aller au sommaire du numéro}

\section{Éditeur(s)}

Université du Québec - INRS-Eau, Terre et Environnement (INRS-ETE)

ISSN

0992-7158 (imprimé)

1718-8598 (numérique)

Découvrir la revue

Citer cet article

Turmel, M.-C., Turgeon, J. M., François, C. \& Cloutier-Hurteau, B. (2005). Saisonnalité du transport de carbone organique dissous dans le ruisseau de l'Hermine, un bassin versant de tête de réseau du Bouclier Canadien. Revue des sciences de l'eau / Journal of Water Science, 18(3), 353-380.

https://doi.org/10.7202/705563ar
Résumé de l'article

Nous avons étudié la variabilité saisonnière de la relation entre les fluctuations des concentrations en carbone organique dissous (COD) dans le ruisseau de l'Hermine (Québec, Canada) et les changements du débit (Q). Un total de 93 événements hydrologiques échantillonnés de 1994 à 2003 et regroupés sur une base saisonnière (hiver-printemps, été, automne) a été analysé. Le modèle de régression linéaire est utilisé afin de déterminer, pour chaque événement, la pente de la relation entre la concentration en COD dans le ruisseau et le débit. Ces pentes sont regroupées par saison et selon un seuil arbitraire de un qui permet de contraster les conditions hydrologiques et climatiques initiales des événements répertoriés. Les résultats du test de Kruskal-Wallis, visant la comparaison entre les événements de pentes supérieures et inférieures à un, montrent clairement la saisonnalité de la relation entre le COD et le débit. La saisonnalité de la relation COD/Q est ensuite mise en relation avec des variables climatiques et hydrologiques susceptibles de conditionner le transport du COD dans le bassin de l'Hermine. Les résultats montrent que les changements saisonniers des conditions climatiques et hydrologiques dans le bassin versant ont un impact significatif sur la relation entre le COD et le débit. Ainsi, le volume de précipitation tombé durant l'événement, la température moyenne de l'air et la température du sol régissent significativement ( $p=0,041 ; 0,001$ et 0,009 respectivement) le transport du COD pour la période hiver-printemps. Les basses températures du sol et l'apport élevé en eau via les précipitations et la fonte favorisent le lessivage intense du COD soluble déjà limité par les basses températures. Au cours de l'été, l'état initial d'humidité du bassin est le principal facteur contrôlant l'évolution des concentrations de COD lors d'une crue; les fortes relations avec le pourcentage d'humidité des sols et le débit total $24 \mathrm{~h}$ avant l'événement le prouvent ( $p=0,039$ et 0,0003 respectivement). Les changements les plus prononcés du COD surviennent, au cours de l'été, suite à une période prolongée de sécheresse. À l'automne, le transport du COD est influencé par le volume de précipitation tombé durant l'événement $(p=0,031)$ et la température du sol $(p=0,042)$. La modélisation de la relation COD/Q par les variables hydro-climatiques montre que $40 \%$ de la relation COD/Q s'explique par la température du sol durant la période d'hiver-printemps. Durant l'été, les conditions initiales d'humidité du bassin, traduites par le débit 24 $\mathrm{h}$ avant l'événement, expliquent à 51\% la relation COD/Q. À l'automne, la relation COD/Q est gouvernée à $50 \%$ à la fois par le volume de précipitation tombé durant l'événement et la température du sol. L'analyse de ces données établit clairement la saisonnalité de la relation COD/Q et que des variables climatiques et hydrologiques permettent de quantifier ces fluctuations saisonnières.
Ce document est protégé par la loi sur le droit d'auteur. L’utilisation des services d'Érudit (y compris la reproduction) est assujettie à sa politique d'utilisation que vous pouvez consulter en ligne.

https://apropos.erudit.org/fr/usagers/politique-dutilisation/ 


\section{Saisonnalité du transport de carbone organique dissous dans le ruisseau de l'Hermine, un bassin versant de tête de réseau du Bouclier Canadien}

\section{Seasonal variations of the transport of dissolved organic carbon in the intermittent stream draining the Hermine headwater catchment on the Canadian Shield}

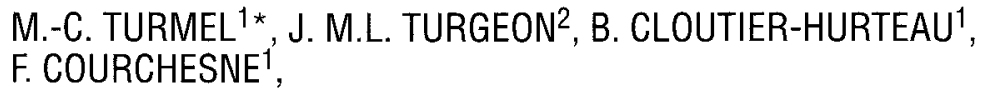

Reçu le 24 octobre 2003, accepté le 21 juin 2005**.

\section{SUMMARY}

The terrestrial organic carbon (C) pool, estimated to $1.5 \times 10^{15} \mathrm{~kg} \mathrm{C}$ for the first meter of soil (AMUNDSON, 2001), represents a major terrestrial elemental stock for which the recycling rate and the response to perturbations are still unknown. Under the present changing climatic conditions, $C$ fluxes in terrestrial ecosystems could be significantly disturbed during the next decades. Indeed, the multi-annual changes in temperature and precipitation are likely to have a major impact on the net primary production and on organic matter decomposition in soils. This situation influences the production of dissolved organic carbon (DOC) in soils, its transport to surface waters and hence, water quality. In this context, a better knowledge of the climatic and hydrologic factors influencing seasonal variations in DOC export is crucial to improve our understanding of the potential transformation of carbon pools and fluxes in terrestrial ecosystems.

The objectives of the present study were 1) to evaluate the seasonality in the relationship between dissolved organic carbon (DOC) concentrations in the stream and streamflow $(Q)$ and 2 ) to quantify the impact of seasonal changes in climatic and hydrological conditions in the watershed on the DOC/Q relationship.

The Hermine catchment is located about $80 \mathrm{~km}$ north of Montréal, Québec, Canada. An intermittent first-order stream drains the 5.1 ha catchment.

1. Département de Géographie, Université de Montréal, CP 6128, Succ. Centre-ville, Montréal, (Québec), H3C 3J7, tél. : (514) 343-8027, fax : (514) 343-8008.

2. Department of Geography, McGill University, Burnside Hall, 805, rue Sherbrooke Ouest, Montréal, Canada, H3A 2K6, têl. : 514-398-4961, fax : 514-398-7437.

* Correspondance: mc.turmel@Umontreal.ca

** Les commentaires seront reçus jusqu'au 30 juin 2006. 
Soils are Orthic and Gleyed Humo-Ferric and Ferro-Humic Podzols. The stream water was sampled daily, from 1994 to 2003 , with an automatic sampler. The stream discharge was calculated from the water level above a $90^{\circ} \mathrm{V}$-notch weir using a Global level sensor bubbler. Soil organic $\mathrm{C}$ content was analysed by the modified Walkley-Black method. The DOC content was estimated by the relationship obtained for eight to ten stream water samples for each biogeochemical compartment analysed with a Shimadzu TOC analyser (Shimadzu, Kyoto, Japan) and the corresponding absorbance measured at $254 \mathrm{~nm}$. From the initial year of the project, 1994, the regression used was $Y=-0.05+32.60 X$ with an $r^{2}$ value of 0.58 and a precision of $0.05 \mathrm{mg} \cdot \mathrm{L}^{-1}$.

The relationship between the DOC concentration and $Q$ at the Hermine was positive and significant $(p<0.01)$ when all data were considered $(n=1960)$. Because of the weakness of this relation $\left(r^{2}=0.12\right)$, the stream samples, from 1994 to 2003, were seasonally split into 93 distinct hydrological events : 33 for winter-spring, 34 for summer and 26 for fall. A linear regression model was used to determine, for each event, the slope of the relationship between the DOC concentrations in the stream and $Q$. To contrast the antecedent conditions of the Hermine watershed, the events from a given season were divided into two groups : events with slope DOC/Q $<1$ and slope DOC/Q $>1$. The Kruskal-Wallis test was then used to establish the link between the slope of the DOC/Q relationships on the one hand, and the environmental watershed conditions on the other hand : the climatic variables (volume of precipitation during event, mean air and soil temperatures) and the hydrological variables (stream discharge $24 \mathrm{~h}$ before the event, soil moisture, and ground water level).

The DOC concentrations in the stream varied on an annual, a seasonal and an event basis. For the period 1994 to 2003 , the annual mean concentrations, calculated from daily samples, varied from 2.0 to $2.5 \mathrm{mg}$ DOC. $\mathrm{L}^{-1}$. On a seasonal basis, mean daily DOC was higher during the summer and the fall (2.9 and $2.8 \mathrm{mg} \mathrm{DOC} \cdot \mathrm{L}^{-1}$ respectively), and lower in the winter-spring (2.1 mg DOC $\left.\cdot \mathrm{L}^{-1}\right)$. The relation between DOC concentrations and $Q$ fluctuated as a function of the seasonal evolution of climatic and hydrological conditions in the Hermine catchment. For winter-spring events, $79 \%$ of the events had a DOC/Q slope lower than one. This period was characterised by high streamflow levels and high total DOC fluxes even though the daily mean DOC concentrations were low $\left(2.1 \mathrm{mg}\right.$ DOC $\left.\cdot \mathrm{L}^{-1}\right)$. The volume of precipitation during the event $(p=0.041)$, the mean air temperature $(p=0.001)$ and the soil temperature $(p=0.009)$ were significantly different between events with slopes lower and higher than one. Indeed the slope of the relation increased when soil temperatures elevated. When the temperatures were higher, DOC export increased and subsurface flow in soil horizon was enriched in DOC. Under colder temperature, the DOC production was limited and the soluble organic substances stored in soils were leached out the catchment with the high volume of precipitation or with the water coming from the snowmelt. For the summer period, there were 20 events with slopes greater than one against 14 with slopes lower than one. The soil humidity $(p=0.039)$ and the total streamflow $24 \mathrm{~h}$ before the event $(p=0.0003)$, were the two variables that significantly distinguished both slope groups. Rapid changes in DOC concentration occured during hydrological events following a long drought period. Under dry conditions, the subsurface flow in soil horizons rich in organic matter, the re-hydration of bed sediments and the hydrophobic behaviour of soil particles could all contribute to the export of high DOC concentrations, even during small events. The relationships between DOC and $Q$, for the fall season, were significantly influenced by the volume of precipitation during the event $(p=0.031)$ and the mean soil temperature $(p=$ 0.042). The events with the lower slopes showed the highest volume of precipitation during the event and the lowest soil temperature. For these events 
occurring under wet conditions, the water originated essentially from the $B$ and $C$ horizons, and DOC fluctuations were then limited because of the low concentrations of $\mathrm{DOC}$ in these horizons (anionic sorption of soluble organic anions by iron oxides).

Best-fit from multiple regressions indicated that $40 \%$ of the link between DOC and $Q$ was explained by the soil temperature during the winter-spring period ( $p=0.0001$ ). For summer, the streamflow $24 \mathrm{~h}$ before the event accounted for $51 \%$ of the variation in $\mathrm{DOC} / \mathrm{Q}$ relationships $(p=0.00001)$. For the fall period, the volume of precipitation during the event and the soil temperature both contributed equally to the $\mathrm{DOC} / \mathrm{Q}$ relationships $(p=$ 0.001 ). From these results, obtained from a multi-year project, it is clear that the relation between $\mathrm{DOC}$ and $\mathrm{Q}$ is a function of the variability in the climatic and hydrological watershed conditions. In a context of global warming, both air and soil temperature will increase. Thus, during winter-spring and fall periods, the duration and the intensity of the DOC production in soils could increase and the export of DOC from the watershed to other surface water system could become more important under equivalent streamflow. Higher air temperature also means higher evapotranspiration by the forest during the summer period, and consequently dryer watershed conditions. $A$ low streamflow and a low soil humidity level could be expected and then, brief rain events could sporadically flush the soluble organic carbon accumulated in the soil. The DOC export would be insignificant for that period, but the DOC concentrations would reach the highest annual level. This new knowledge on the DOC/Q relationships, at the hydrological event scale of the possible effects of global warming on the carbon cycle in forested ecosystems increase our understanding.

Keywords : dissolved organic carbon, solute, stream, forested watershed, seasonality.

\section{RÉSUMÉ}

Nous avons étudié la variabilité saisonnière de la relation entre les fluctuations des concentrations en carbone organique dissous (COD) dans le ruisseau de l'Hermine (Québec, Canada) et les changements du débit $(Q)$. Un total de 93 événements hydrologiques échantillonnés de 1994 à 2003 et regroupés sur une base saisonnière (hiver-printemps, été, automne) a été analysé. Le modèle de régression linéaire est utilisé afin de déterminer, pour chaque événement, la pente de la relation entre la concentration en COD dans le ruisseau et le débit. Ces pentes sont regroupées par saison et selon un seuil arbitraire de un qui permet de contraster les conditions hydrologiques et climatiques initiales des événements répertoriés. Les résultats du test de Kruskal-Wallis, visant la comparaison entre les événements de pentes supérieures et inférieures à un, montrent clairement la saisonnalité de la relation entre le COD et le débit. La saisonnalité de la relation COD/Q est ensuite mise en relation avec des variables climatiques et hydrologiques susceptibles de conditionner le transport du COD dans le bassin de l'Hermine. Les résultats montrent que les changements saisonniers des conditions climatiques et hydrologiques dans le bassin versant ont un impact significatif sur la relation entre le COD et le débit. Ainsi, le volume de précipitation tombé durant l'événement, la température moyenne de l'air et la température du sol régissent significativement $(p=0,041 ; 0,001$ et 0,009 respectivement) le transport du COD pour la période hiver-printemps. Les basses températures du sol et l'apport élevé en eau via les précipitations et la fonte favorisent le lessivage intense du COD soluble déjà limité par les basses températures. Au cours de l'été, l'état initial d'humidité du bassin est le principal facteur contrôlant l'évolution des concentrations de COD lors d'une crue; les fortes relations avec le pourcentage d'humidité des sols et le débit total 24 h avant l'événe- 
ment le prouvent $(p=0,039$ et 0,0003 respectivement). Les changements les plus prononcés du COD surviennent, au cours de l'été, suite à une période prolongée de sécheresse. À l'automne, le transport du COD est influencé par le volume de précipitation tombé durant l'événement $(p=0,031)$ et la température du sol $(p=0,042)$. La modélisation de la relation COD/Q par les variables hydro-climatiques montre que $40 \%$ de la relation COD/Q s'explique par la température du sol durant la période d'hiver-printemps. Durant l'été, les conditions initiales d'humidité du bassin, traduites par le débit 24 h avant l'événement, expliquent à $51 \%$ la relation COD/Q. À l'automne, la relation COD/Q est gouvernée à $50 \%$ à la fois par le volume de précipitation tombé durant l'événement et la température du sol. L'analyse de ces données établit clairement la saisonnalité de la relation COD/Q et que des variables climatiques et hydrologiques permettent de quantifier ces fluctuations saisonnières.

Mots clés : carbone organique dissous, transport en solution, ruisseau, bassin versant forestier, saisonnalité.

\section{1 - INTRODUCTION}

Les évaluations récentes de contenu global en carbone $(\mathrm{C})$ organique dans les sols sont généralement convergentes et montrent que cette réserve atteint $1,5 \times 10^{15} \mathrm{~kg} \mathrm{C}$ dans le premier mètre du sol (JOBBÁGY et JACKSON, 2000; KIRSCHBAUM, 2000; AMUNDSON, 2001). II s'agit d'un stock élémentaire terrestre majeur dont la vitesse de recyclage et la réponse aux perturbations restent méconnues. Le $\mathrm{C}$ organique est aussi stocké dans les divers compartiments biogéochimiques des écosystèmes terrestres, par exemple au niveau de la végétation ou des sédiments. Entre ces compartiments, il est transporté en phase liquide ou particulaire. Dans la phase dissoute, il se présente en particulier sous la forme de substances humiques et d'acides organiques de faible masse moléculaire (VAN CLEVE et POWERS, 1995). Ces flux de carbone organique dissous (COD) constituent une source de $\mathrm{C}$ pour les microorganismes et contribuent, entre autres, à la mobilisation et à la complexation des nutriments et des métaux traces ainsi qu'à leur transport vers les eaux souterraines ou de surface, à la formation des sols, à l'altération des minéraux et au transfert d'acidité dans les sols (STROBEL et al., 2001).

La production et le transport du COD dans les écosystèmes terrestres subissent l'influence d'une gamme de facteurs environnementaux, tels que le régime pluviométrique et thermique, la physiographie du bassin, le type de végétation et de microorganismes, l'abondance des sols organiques et l'hydrologie du sol (ECKHARDT et MOORE, 1990; DALVA et MOORE, 1991; KALBITZ et al., 2000). Dans un contexte de changements climatiques, le bilan du $C$ dans les écosystèmes terrestres risque d'être particulièrement perturbé au cours des prochaines décennies. En effet, les changements pluriannuels des températures et des précipitations ont un impact majeur sur l'évolution de l'équilibre entre le taux de production primaire nette de biomasse et le taux de décomposition de la matière organique au sol et donc, sur la production du COD qui sera subséquemment transporté vers les eaux de surface (SCHIMEL et al., 2001). 
Diverses études en laboratoire (CHRIST et DAVID, 1996) et sur le terrain (KIRSCHBAUM, 1995) ont été réalisées sur l'effet des variations de température sur la production de COD. Ces travaux ont montré qu'une hausse de la température du sol entraîne une augmentation de l'activité biologique, notamment dans les horizons organiques, augmentation qui se traduit par une plus grande production de COD dans les sols. Le régime pluviométrique contrôle également les concentrations de COD dans les solutions de sols et de ruisseaux. Sur ce dernier point, la nature des observations varie selon l'échelle temporelle considérée. À l'échelle saisonnière, plus les apports en eau dans le bassin versant sont abondants, plus le temps de contact moyen entre l'eau de percolation et le sol diminue, et plus les concentrations de COD dans le ruisseau sont faibles (MULHOLLAND et HILL, 1997; QUALLS et al., 2002). À l'échelle événementielle, plusieurs études ont, par contre, montré une relation positive entre le débit du cours d'eau et la concentration de COD (BUFFAM et al., 2001). L'évolution des changements dans le cheminement de l'eau dans le sol en cours d'événement est souvent invoquée afin d'expliquer ce contraste. La production de COD est également associée au contenu en eau du sol (BORKEN et al., 1999). En effet, plusieurs études de laboratoire soulignent que l'activité microbienne et la décomposition de la matière organique demeurent relativement constantes pour une large gamme de taux d'humidité (SKOPP et al., 1990). Toutefois, au-delà de certains seuils, donc dans des sols trop secs ou trop humides, l'impact du contenu en eau sur l'activité microbienne devient déterminant et la production de COD s'en trouve réduite. Par la suite, la réhydratation de sols secs entraîne souvent de fortes hausses des concentrations en COD (PRETCHEL et al., 2000).

Au plan des variations saisonnières de la production et du transport du COD dans le sol, DOSSKEY et BERTSCH (1997), en Caroline du Nord, n'ont pu observer d'effet sur les concentrations de COD à cause des contrastes climatiques saisonniers trop faibles. BUFFAM et al. (2001) notent qu'il n'y a aucune différence saisonnière dans les concentrations de COD durant l'écoulement d'étiage du ruisseau, mais que la composition du COD peut varier d'une saison à l'autre. Cependant, HONGVE (1999) a mesuré des pics de COD en automne et suggère que cette saisonnalité des concentrations de COD est liée au lessivage de la litière fraîche. Des concentrations maximales de COD ont aussi été enregistrées durant la période estivale par TIPPING et al. (1999), surtout après de longues sécheresses.

Compte tenu des importantes masses de $\mathrm{C}$ organique stockées dans les sols, et de l'incertitude quant à la magnitude et la direction de l'effet des changements des conditions climatiques sur les bilans en $\mathrm{C}$ dans les sols, il importe donc d'étudier en détail la nature des variations temporelles des flux en COD dans les sols et les cours d'eau afin d'apprécier le spectre des conditions possibles. L'amélioration de notre connaissance des facteurs climatiques et hydrologiques influençant les variations saisonnières actuelles de la production de COD dans les sols et de son transport dans les cours d'eau assurera une meilleure compréhension des transformations potentielles des stocks et des flux en $\mathrm{C}$ dans les écosystèmes terrestres. Cette situation est particulièrement critique pour les bassins versants forestiers des milieux nordiques où les Podzols dominent puisque les flux de COD peuvent constituer une fraction importante des pertes en C dans ces sols (CRONAN et AIKEN, 1985; NEFF et ASNER, 2001). 
Dans ce contexte, les objectifs de la présente étude sont : 1) d'établir la saisonnalité de la relation entre les variations des concentrations en carbone organique dissous (COD) dans le ruisseau et les fluctuations du débit (Q) et 2) de quantifier l'impact des changements saisonniers des conditions climatiques et hydrologiques dans le bassin versant sur la relation entre COD et $Q$.

\section{2 - MÉTHODOLOGIE}

\subsection{Terrain d'étude}

Le bassin versant de l'Hermine est situé à la Station de Biologie des Laurentides (SBL) de l'Université de Montréal à $80 \mathrm{~km}$ au nord de Montréal, Québec, Canada $\left(45^{\circ} 59^{\prime} \mathrm{N}, 74^{\circ} 01^{\prime} \mathrm{W}\right.$, altitude de $400 \mathrm{~m}$ ) (figure 1a). La moyenne annuelle des précipitations est d'environ $1100 \mathrm{~mm}$ dont $30 \%$ tombe sous forme de neige (figure $1 \mathrm{~b}$ ). Un ruisseau intermittent de tête de réseau draine ce bassin d'une superficie de 5,1 ha (figure 1c). Le substrat rocheux est composé d'anorthosite du Précambrien de la série de Morin (MCGERRIGLE, 1976) lequel est recouvert d'un till de fond d'environ un à deux mètres d'épaisseur. Des podzols ferro-humiques et humo-ferriques gleyifiés ou orthiques se développent dans ce matériel d'origine (Comité d'experts sur la prospection pédologique d'Agriculture Canada, 1987). Des détails sur la composition chimique des sols sont disponibles dans COURCHESNE et al. (2001). Le couvert végétal est composé de $78 \%$ (pourcentage de la surface terrière) d'érable à sucre (Acer saccharum Marsh.), 9 \% de hêtre à grandes feuilles (Fagus grandifolia Ehrn.) et $6 \%$ de bouleau jaune (Betula alleghaniensis Britton). La forêt a été coupée ou brûlée au début du siècle dernier (BÉLANGER et al., 2002).

\subsection{Données climatiques et hydrologiques}

Le débit du ruisseau a été mesuré en continu à la station de jaugeage (figure 1c) à l'aide d'un capteur de niveau d'eau bulle à bulle. La température de l'air et le niveau des précipitations incidentes ont été obtenus sur une base quotidienne à la station météorologique du ministère de l'Environnement du Québec situé sur le territoire de la SBL à environ $1 \mathrm{~km}$ du bassin de l'Hermine. En 1993, neuf parcelles de $300 \mathrm{~m}^{2}$ reflétant l'hétérogénéité spatiale du couvert végétal, des sols et de la topographie ont été définies (figure 1c). À chacune de ces parcelles, le volume des pluviolessivats, le niveau de la nappe phréatique, le pourcentage d'humidité du sol ainsi que la température du sol ont été mesurés toutes les deux semaines pendant la saison de croissance (mi-mai à minovembre) et une fois par mois le reste de l'année. Les pluviolessivats ont été récoltés dans des collecteurs $\left(500 \mathrm{~cm}^{2}\right)$ installés à $1,5 \mathrm{~m}$ de hauteur et reliés à un contenant enfoui dans le sol. En hiver, ils ont été remplacés par des collecteurs à neige d'une surface de $50 \mathrm{~cm}^{2}$. Le niveau de la nappe phréatique a été mesuré dans des puits d'accès avec un senseur de conductivité. Le contenu en eau du sol a été estimé à une profondeur de $25 \mathrm{~cm}$ à l'aide de capteurs installés en permanence permettant de mesurer la réflectométrie dans le domaine temporel (TDR : Time Domain Reflectrometry). Cette mesure de l'humidité n'a 
pas été effectuée en hiver. La température des sols a été obtenue à une profondeur de $15 \mathrm{~cm}$, dans l'horizon B supérieur. Les flux en eau dans les divers horizons de sols ont été calculés selon ARP et YIN (1992).
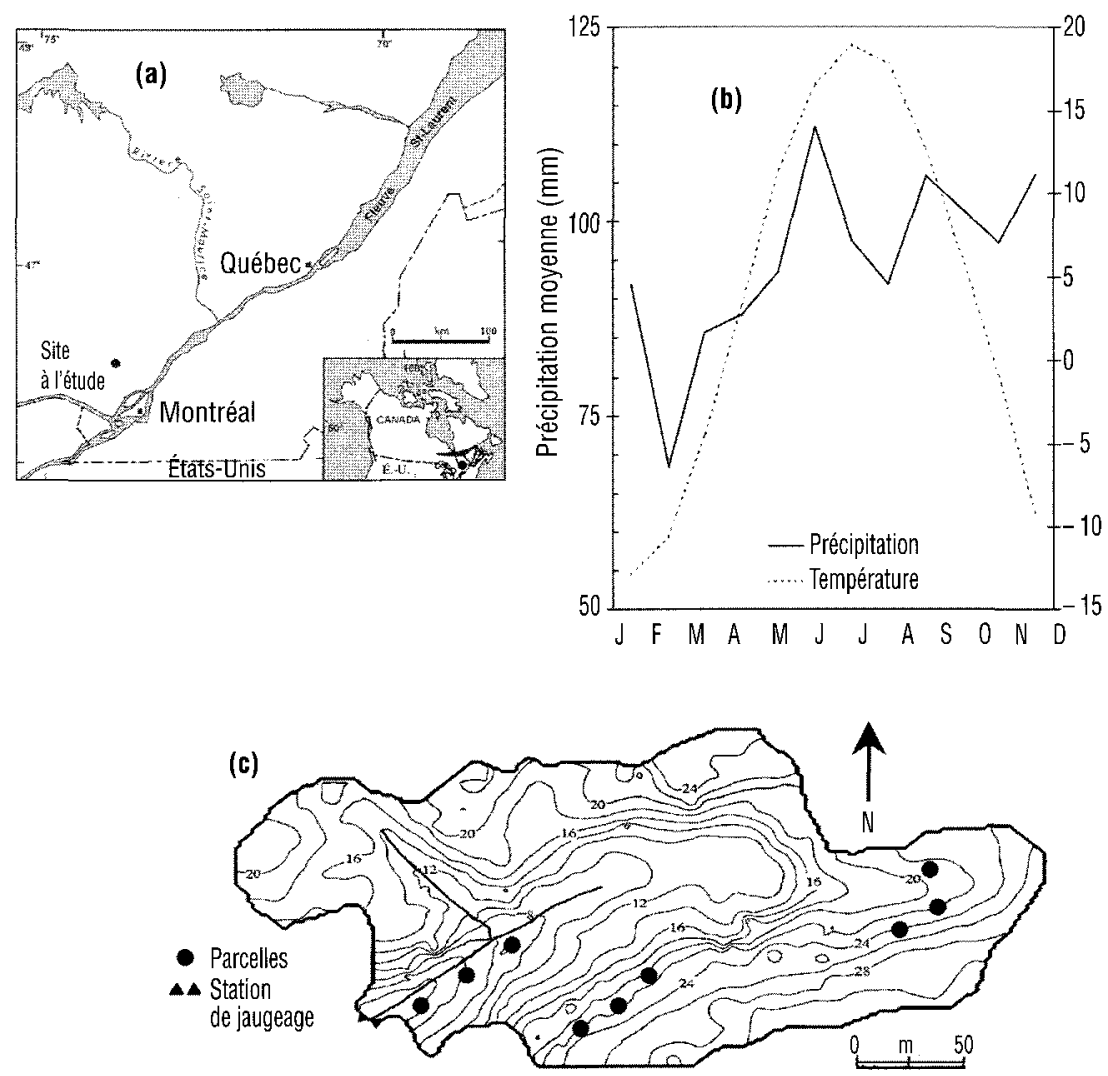

Figure 1 (a) Localisation du bassin versant de l'Hermine, (b) moyennes mensuelles des précipitations et des températures de l'air à l'Hermine au cours de la période 1973 à 2002 et (c) carte de l'Hermine incluant la position des neuf parcelles et de la station de jaugeage (élévation en mètres au-dessus de la station de jaugeage).

(a) Location of the Hermine catchment, (b) mean monthly precipitation and air temperature for the catchment from 1973 to 2002, and (c) map of the Hermine including the nine plots and the weir (elevation in meters above the weir). 


\subsection{Prélèvement des solutions et des sols}

L'eau du ruisseau destinée à l'analyse du COD a été récoltée quotidiennement par un échantillonneur automatique pour les années 1994 à 2003. II est à noter qu'aucune donnée n'est disponible pour l'année 1998. L'ensemble de ces solutions a été utilisé afin d'y sélectionner les événements hydrologiques pour lesquels la réponse du COD est significative. Le découpage des hydrogrammes a ainsi permis de sélectionner 93 événements hydrologiques pour lesquels la réponse du COD était significative à un changement de débit $(\alpha=0,05)$. Le nombre de points retenus pour chacun des événements variait entre quatre et 30 selon l'étendue de l'hydrogramme de crue. Ces 93 événements ont été regroupés sur une base saisonnière en suivant les dates calendriers. Cette division des saisons permettait d'éviter toute interprétation subjective liée aux conditions édaphiques, phéonologiques ou biogéochimiques du bassin et leur effet possible sur le COD. Ainsi la période de l'hiverprintemps va du 21 décembre au 20 juin, l'été s'étend du 21 juin au 20 septembre et l'automne du 21 septembre au 20 décembre. Les saisons d'hiver et de printemps ont été groupées, car durant la période hivernale les événements hydrologiques sont quasi inexistants étant donné les températures froides. Lorsque des événements hydrologiques surviennent en hiver, ils sont liés à un redoux qui provoque une période de fonte ponctuelle similaire aux fontes printanières.

Les précipitations incidentes, les pluviolessivats et les solutions de sol ont été prélevés sur une base bimensuelle pendant la saison de croissance (mi-mai à mi-novembre) et une fois par mois le reste de l'année. Les solutions de sols ont été récoltées à l'aide de lysimètres sans tension logés sous la litière forestière ainsi qu'à 25 et $50 \mathrm{~cm}$ de profondeur, dans l'horizon B podzolique. Les horizons de 15 profils de sols ont été échantillonnés en août 1986. La densité des horizons a été estimée par gravimétrie. Les sols ont été séchés à l'air et tamisés à 2-mm avant l'analyse du C organique.

\subsection{Analyses chimiques}

Le contenu en $\mathrm{C}$ organique de la phase solide des sols a été analysé selon la méthode modifiée de Walkley-Black (TIESSEN et MOIR, 1993). Le carbone organique dissous a été estimé à partir de la relation obtenue entre les concentrations en COD mesuré sur un analyseur de carbone Shimadzu (Shimadzu, Kyoto, Japon) et les mesures d'absorbance à $254 \mathrm{~nm}$ (MOORE, 1985). Les mesures d'absorbance-254 nm ont été effectuées sur les solutions filtrées à $0,4 \mu \mathrm{m}$; ce sont les mêmes que celles utilisées pour l'analyse réelle de COD. La relation Abs-254/COD a été calculée à partir de 8 à 10 échantillons pour chacun des compartiments biogéochimiques du bassin de l'Hermine. Le tableau 1 présente l'ensemble des paramètres obtenus. L'équation utilisée pour convertir les valeurs d'absorbance en concentrations de COD pour le ruisseau $(\mathrm{Y}=-0,05+32,60 \mathrm{X})$ a été élaborée à partir de l'analyse réelle du COD de huit échantillons couvrant les quatre saisons. Ainsi, l'équation résultante tient compte de la variabilité annuelle dans la composition du $\mathrm{C}$ organique. Les résultats de COD obtenus de cette conversion sont des valeurs estimées du COD pour le bassin de l'Hermine, avec une précision de $0,05 \mathrm{mg} \cdot \mathrm{L}^{-1}$. L'erreur sur la moyenne pour les différents compartiments est similaire à celle dispo- 
nible dans la littérature pour une méthode similaire (MOORE, 1987). Les interférences liées au fer libre sont négligeables étant donné le $\mathrm{pH}$ moyen du ruisseau qui se situe aux environs de 6,20.

Tableau 1 Paramètres des régressions obtenues entre l'absorbance des solutions à $254 \mathrm{~nm}$ et les concentrations mesurées en carbone organique dissous à l'aide d'un analyseur de carbone.

Table 1 Parameters obtained from the regressions between absorbance at $254 \mathrm{~nm}$ and the dissolved organic carbon concentrations measured with the carbon analyser.

\begin{tabular}{|c|c|c|c|c|c|c|c|c|}
\hline $\begin{array}{l}\text { Compartiment } \\
\text { biogéochimique }\end{array}$ & $\mathrm{n}$ & $\begin{array}{c}\text { COD } \\
\text { moyen } \\
\left(m g \cdot L^{-1}\right)\end{array}$ & $\begin{array}{l}\text { Étendue } \\
\text { des valeurs } \\
\text { de COD } \\
\left(\mathrm{mg} \cdot \mathrm{L}^{-1}\right)\end{array}$ & $\begin{array}{c}\text { Penie } \\
\text { de la relation } \\
\text { ABS- } 254 / \mathrm{COD} \\
\left.\text { (abs/mg } \cdot \mathrm{L}^{-1}\right)\end{array}$ & $\begin{array}{l}\text { Ordonnée } \\
\text { à l'origine } \\
\left.\text { (mg. } \mathrm{L}^{-1}\right)\end{array}$ & $r^{2}$ & $\begin{array}{l}\text { Erreur } \\
\text { type }\end{array}$ & $\begin{array}{c}\text { Erreur } \\
\text { sur la moyenne } \\
\%\end{array}$ \\
\hline Précipitation & 10 & 5,74 & $2,05-10,71$ & 31,31 & 3,02 & 0,94 & 1,05 & 19 \\
\hline Pluviolessivat & 10 & 11,53 & $4,66-31,43$ & 39,18 & $-1,49$ & 0,85 & 3,33 & 29 \\
\hline Solution de sol à $0 \mathrm{~cm}$ & 9 & 26,44 & $13,30-33,50$ & 18,99 & 3,60 & 0,93 & 1,64 & 6 \\
\hline Solution de sol à $50 \mathrm{~cm}$ & 10 & 2,53 & $1,79-3,80$ & 21,78 & 1,28 & 0,73 & 0,31 & 13 \\
\hline Ruisseau & 10 & 2,90 & $2,41-4,31$ & 32,60 & $-0,05$ & 0,58 & 0,37 & 13 \\
\hline Neige & 8 & 1,52 & $1,19-2,02$ & 14,36 & 1,17 & 0,75 & 0,14 & 10 \\
\hline
\end{tabular}

a : Mesure directe sur analyseur de carbone (Shimadzu, Kyoto, Japon).

\subsection{Analyses statistiques}

Le modèle de régression linéaire des moindres carrés a été utilisé afin de déterminer, sur une base événementielle, la pente de la relation entre la concentration en COD dans le ruisseau et le débit. Tous les événements présentant des régressions significatives $(\alpha=0,05)$ ont été retenus pour l'analyse du lien qui existe entre le COD et le Q. L'observation des graphiques des 93 événements hydrologiques retenus a permis de distinguer deux pôles de réponse du COD à un changement de débit : de fortes réponses du COD suite à une faible variation du débit et, à l'opposé, des variations faibles de COD pour de forts débits (figure 4a). Afin de mieux cerner les conditions environnementales expliquant cette bipolarité de la relation COD/Q, une limite de pente égale à un a été fixée. Cette limite n'a aucune signification biogéochimique; elle est toutefois utile pour établir le contraste dans les conditions hydro-climatiques entre les deux groupes d'événements. Ainsi, une gamme de variables hydrologiques (débit $24 \mathrm{~h}$ avant événement, l'humidité antécédente du sol, niveau de la nappe phréatique) et climatiques (volume des précipitations durant l'événement, température de l'air et température du sol), traduisant l'état du système au moment des événements, ont été comparés pour les deux groupes de pentes (pente COD/Q $<1$ et pente $C O D / Q>1$ ) et pour chacune des saisons.

Le recours à des tests statistiques non paramétriques a été nécessaire compte tenu du nombre élevé de distributions qui ne respectaient pas le postulat de normalité pour les variables hydrologiques et climatiques. Le test sur la moyenne de Kruskal-Wallis a été utilisé afin d'établir des contrastes hydro-climatiques entre les deux groupes d'événements. Ce test indique une différence significative entre les deux groupes lorsque la valeur de $p$ est inférieure à 0,05 . 
Ces tests ont d'abord été effectués sur l'ensemble des événements, sans égard aux saisons, et pour chacune des saisons (hiver-printemps, été et automne) afin d'établir la saisonnalité de la relation COD/Q.

Des analyses de régressions multiples ont finalement été effectuées pour chacune des saisons afin de déterminer la ou les variables climatiques et hydrologiques expliquant le mieux le lien qui existe entre le COD et le Q. La version 11.5 de SPSS (2003) a été utilisée pour la conduite de ces tests.

\section{3 - RÉSULTATS ET DISCUSSION}

\subsection{Contenu en $\mathrm{C}$ organique des sols et flux de COD dans le bassin versant}

Dans les sols du bassin de l'Hermine, les horizons organiques L, F et $\mathrm{H}$ présentent les concentrations et les contenus en $C$ organique les plus élevés (tableau 2). Toutefois, les horizons B podzoliques (Bhf et Bf) constituent la réserve la plus importante de $\mathrm{C}$ organique en raison de leur épaisseur et de leur plus grande densité. Les concentrations de $C$ diminuent ensuite rapidement des horizons $\mathrm{B}$ vers le matériel d'origine. La présence de deux zones d'accumulation de matière organique dans le profil de ces sols résulte de la podzolisation. Le contenu moyen en $\mathrm{C}$ organique des sols de l'Hermine totalise $457 \mathrm{Mg} \mathrm{C} \cdot \mathrm{ha}^{-1}$; il se chiffre à $345 \mathrm{Mg} \mathrm{C} \cdot \mathrm{ha}^{-1}$ pour le premier mètre de sol minéral, une valeur similaire à celle des sols de milieux comparables (AMUNDSON, 2001). Le contenu en matière organique des sols a souvent un impact sur la concentration de COD dans le ruisseau. Par exemple, AITKENHEAD et al. (1999) ont montré qu'il existe une forte relation entre la magnitude des réservoirs de $\mathrm{C}$ organique dans le sol et la concentration de COD dans le cours d'eau pour des bassins versants couvrant une gamme d'échelles spatiales. Cette relation est particulièrement marquée pour des bassins de petite superficie $\left(<5 \mathrm{~km}^{2}\right)$ et elle est suffisamment prononcée pour servir d'outil de prédiction de l'effet des variations du stock de $C$ dans les sols sur les concentrations de COD dans les ruisseaux.

Les concentrations relatives du COD varient en fonction du compartiment biogéochimique (tableau 3). Les précipitations présentent une concentration moyenne de $4,1 \mathrm{mg} \cdot \mathrm{L}^{-1}$. Cette moyenne est légèrement supérieure à celle retrouvée pour des bassins versants similaires. Ceci s'explique en partie par le type de collecteur (dépôts sec et humide), la fréquence de d'échantillonnage (à toutes les 2 ou 4 semaines selon les saisons) et les conditions d'entreposage sur le terrain. Les concentrations de COD augmentent dans le pluviolessivat pour atteindre un maximum sous les horizons organiques puis diminuent abruptement dans les horizons minéraux du sol et le ruisseau. Les flux en COD suivent la même tendance et montrent un bilan annuel positif signalant une forte rétention de COD dans le bassin. Ces tendances s'apparentent à celles généralement observées dans des écosystèmes forestiers du nord-est de l'Amérique du Nord, d'Europe et de Scandinavie (HINTON et al., 1998; SOLINGER et al., 2001). Les fortes concentrations en COD mesurées sous la litière forestière sont associées au lessivage des produits de la décomposition de la 
matière organique et aux flux en COD dans les pluviolessivats. La baisse marquée des concentrations en COD sous les horizons organiques résulte de l'adsorption anionique par ces sols (COURCHESNE et HENDERSHOT, 1989) et par les horizons $B$ podzoliques en général (MCDOWELL et LIKENS, 1988). La hausse du contenu en $\mathrm{C}$ organique dans les horizons Bhf et Bf en témoigne (tableau 2).

Tableau 2 Concentrations et contenus moyens de carbone organique dans les horizons de 15 profils de sols du bassin de l'Hermine. Les écarts types sont présentés entre parenthèses. Les données sont tirées de Savoie (1988) et de COURCHESNE et al. (2002).

Table 2 Mean organic carbon concentrations and contents in the horizons of 15 soil profiles of the Hermine watershed. Standard deviations are in parentheses. The data are from Savoie (1988) and COURCHESNE et al. (2002).

\begin{tabular}{ccccc}
\hline Horizon & $\begin{array}{c}\text { Profondeur } \\
\text { moyenne } \\
\mathbf{c m}\end{array}$ & $\begin{array}{c}\text { Densité } \\
\mathbf{k g} \cdot \mathbf{m}^{-3}\end{array}$ & $\begin{array}{c}\text { Concentration } \\
\mathbf{C} \text { organique } \\
\mathbf{g} \cdot \mathbf{k g}^{-1}\end{array}$ & $\begin{array}{c}\text { Contenu } \\
\text { Corganique } \\
\text { par horizon } \\
\mathbf{M g} \cdot \mathbf{h a} \mathbf{-}^{-1}\end{array}$ \\
\hline $\mathrm{L}$ & $11-8$ & 190 & $510(77)$ & $13,8(2,08)$ \\
$\mathrm{F}$ & $8-4$ & 180 & $490(74)$ & $35,3(5,33)$ \\
$\mathrm{H}$ & $4-0$ & 270 & $481(72)$ & $51,9(7,78)$ \\
$\mathrm{Ae}$ & $0-2$ & 1190 & $48(7,2)$ & $11,4(1,71)$ \\
$\mathrm{Bhf1}$ & $2-12$ & 610 & $129(19)$ & $78,7(11,59)$ \\
$\mathrm{Bhf}$ & $12-41$ & 790 & $67(10)$ & $153,5(22,91)$ \\
$\mathrm{Bf}$ & $41-63$ & 890 & $43(6,4)$ & $84,2(12,53)$ \\
$\mathrm{BCg}$ & $63-80$ & 1110 & $6(0,9)$ & $11,3(1,70)$ \\
$\mathrm{Cg}$ & $80-110$ & 1420 & $4(0,2)$ & $17,0(0,85)$ \\
\hline
\end{tabular}

Tableau 3 Concentrations et flux moyens de carbone organique dissous (COD) dans différents compartiments biogéochimiques de l'écosystème de l'Hermine pour la période de 1994 à 1999. Les écarts types sont présentés entre parenthèses.

Table 3 Dissolved organic carbon (DOC) concentrations and fluxes for different biogeochemical compartments of the Hermine ecosystem for the period 1994 to 1999. Standard deviations are in parentheses.

\begin{tabular}{ccc}
\hline Compartiment biogéochimique & $\begin{array}{c}\text { Concentration } \\
\mathbf{C O D} \\
\mathbf{m g} \cdot \mathrm{L}^{-\mathbf{1}}\end{array}$ & $\begin{array}{c}\text { Flux } \\
\mathbf{C O D} \\
\mathbf{k g} \cdot \mathbf{h a}^{-1} \cdot \mathbf{a n}^{-\mathbf{1}}\end{array}$ \\
\hline Précipitation & $4,1(0,84)$ & $45,6(3,6)$ \\
Pluviolessivat & $9,4(0,36)$ & $93,1(48,9)$ \\
Solution de sol à 0 cm & $28,8(10,55)$ & $235(44,7)$ \\
Solution de sol à $25 \mathrm{~cm}$ & $3,0(2,31)$ & $38,2(9,5)$ \\
Solution de sol à $50 \mathrm{~cm}$ & $2,5(0,92)$ & $16,9(3,9)$ \\
Ruisseau & $2,5(1,22)$ & $16,3(7,7)$ \\
\hline
\end{tabular}




\subsection{Variations temporelles des concentrations en COD dans le ruisseau}

Les concentrations relatives en COD dans le ruisseau de l'Hermine fluctuent sur une base annuelle, saisonnière et événementielle. Pour la durée du projet, soit de 1994 à 2003, cinq années présentent des séries complètes de mesures quotidiennes de débit et de COD (tableau 4). À l'échelle annuelle, ces années ont des concentrations moyennes relatives non pondérées variant de 2,1 à $2,5 \mathrm{mg}$ COD $\cdot \mathrm{L}^{-1}$. Ces données sont semblables aux valeurs observées dans plusieurs autres petits cours d'eau du nord-est de l'Amérique du Nord (MCDOWELL et LIKENS, 1988). Des variations saisonnières sont aussi notables. Les concentrations moyennes relatives du COD sont maximales en été et à l'automne (2,9 et $2,8 \mathrm{mg} \cdot \mathrm{L}^{-1}$, respectivement) et minimales durant l'hiver-printemps $\left(2,1 \mathrm{mg} \cdot \mathrm{L}^{-1}\right)$ (tableau 4). Finalement, des écarts marqués de concentration allant

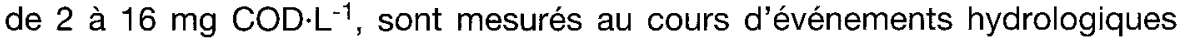
individuels. Ces variations en COD sont généralement synchrones avec les fluctuations du débit, comme le montre la figure 2 pour les années 2002 et 2003.

Tableau 4 Intrants et extrants totaux moyens en eau, température moyenne de l'air, concentration moyenne quotidienne en carbone organique dissous (COD) et flux total moyen de COD dans le ruisseau du bassin de l'Hermine sur une base saisonnière et annuelle.

Table 4 Mean total water inputs and outputs, average air temperature, daily mean dissolved organic carbon (DOC) concentration and mean total DOC flux in the stream of the Hermine watershed on a seasonal and annual basis.

\begin{tabular}{ccccc}
\hline Propriétés & $\begin{array}{c}\text { Hiver- Printemps } \\
(\mathbf{n = 1 8 2})^{\mathrm{a}}\end{array}$ & $\begin{array}{c}\text { Été } \\
(\mathbf{n = 9 2})\end{array}$ & $\begin{array}{c}\text { Automne } \\
(\mathbf{n}=\mathbf{9 1})\end{array}$ & $\begin{array}{c}\text { Année } \\
(\mathbf{n}=\mathbf{3 6 5})\end{array}$ \\
\hline Précipitation totale $(\mathrm{mm})^{\mathrm{b}}$ & $530(53)$ & $315(71)$ & $351(123)$ & $1197(116)$ \\
Débit total $(\mathrm{mm})^{\mathrm{c}}$ & $359(101)$ & $12(11)$ & $129(122)$ & $499(189)$ \\
Température moyenne air $\left({ }^{\circ} \mathrm{C}\right)^{\mathrm{b}}$ & $-0,8(1,1)$ & $17,9(1,0)$ & $1,5(1,6)$ & $4,5(0,8)$ \\
Concentration moyenne COD $\left(\mathrm{mg} \cdot \mathrm{L}^{-1}\right)^{\mathrm{c}}$ & $2,1(0,2)$ & $2,9(0,3)$ & $2,8(0,5)$ & $2,4(0,2)$ \\
Flux totai COD ruisseau $\left(\mathrm{kg} \cdot \mathrm{ha}^{-1}\right)^{\mathrm{c}}$ & $9,5(2,6)$ & $0,4(0,5)$ & $5,5(6,2)$ & $15,5(8,0)$ \\
\hline
\end{tabular}

$\mathrm{a}: \mathrm{n}=$ nombre de jours par période.

$\mathrm{b}:$ Moyennes et écarts types portant sur les neuf années de l'étude.

c: Moyennes et écarts types portant sur les années pour lesquelles les débits enregistrés et l'échantillonnage du ruisseau sont complets (1995-1996-1997-2002-2003). 


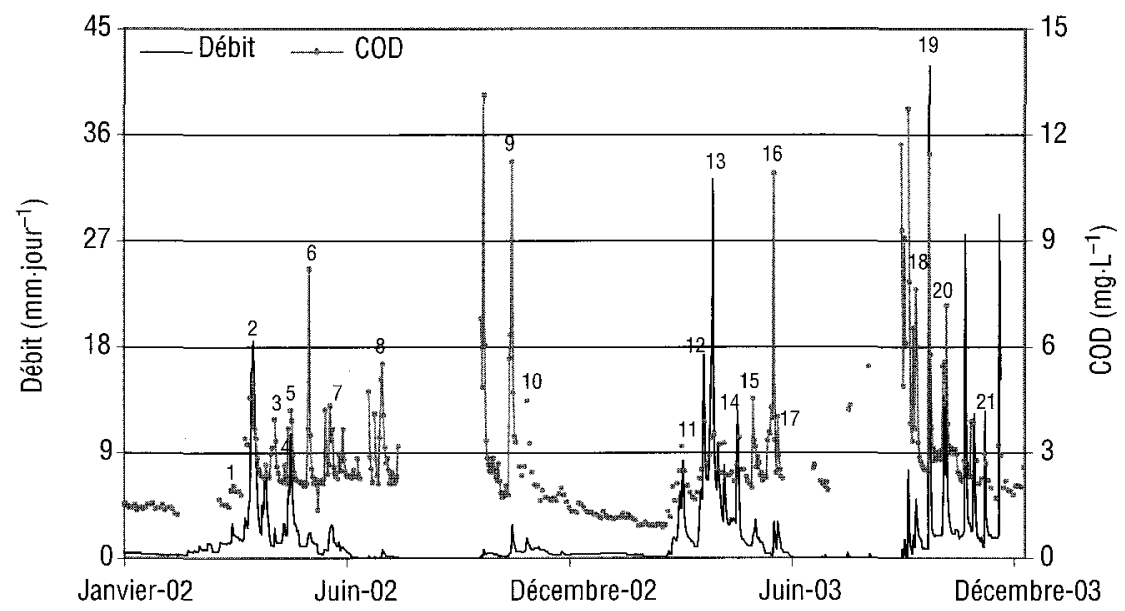

Figure 2 Concentration de carbone organique dissous (COD) et débit (Q) instantané pour le bassin de l'Hermine en 2002 et 2003. Les chiffres 1 à 21 identifient les événements hydrologiques où la relation entre COD et $Q$ est significative.

Dissolved organic carbon (DOC) concentrations and streamflow (Q) in the Hermine catchment for 2002 and 2003. The numbers 1 to 21 represent hydrological events with a significant relationship between $D O C$ and $Q$.

\subsection{Relation entre la concentration en COD et le débit du ruisseau}

À l'échelle annuelle, et pour les années dont le débit enregistré est complet, le débit du ruisseau de l'Hermine représente en moyenne $42 \%$ des dépôts en eau sur le bassin (tableau 4). Cette proportion est minimale à $30 \%$ en 1995 et atteint un sommet à $57 \%$ en 2003. Sur une base saisonnière, seulement $3 \%$ des précipitations sont converties en débit à l'exutoire au cours de l'été alors que $37 \%$ le sont pour l'automne et $70 \%$ pour la période de l'hiver et du printemps (21 décembre au 20 juin), période pendant laquelle la température moyenne de l'air se maintient sous le point de congélation (tableau 4).

Dans l'Hermine, la relation entre le débit quotidien et la concentration en COD dans le ruisseau est positive et significative (figure 3 ) lorsque toutes les données de la période 1994-2003 sont considérées $(n=1960)$. Cette relation est néanmoins faible $\left(r^{2}=0,12\right)$ et présente une très forte dispersion des valeurs. MCDOWELL et WOOD (1984) ont aussi montré que la concentration de COD dans le ruisseau était significativement mais faiblement liée au débit [COD $\left.\left(\mathrm{mg} \cdot \mathrm{L}^{-1}\right)=1,17 \mathrm{Q}\left(\mathrm{L} \cdot \mathrm{sec}^{-1}\right)+0,064, \mathrm{r}^{2}=0,46, p<0,05\right]$ dans le bassin de Hubbard Brook. En ce sens, la figure 3 ne reflète pas adéquatement la relation fine qui existe entre la concentration de COD et le débit observable, sur une base événementielle, dans l'Hermine (figure 2) et dans d'autres cours d'eau de milieux forestiers tempérés (BUFFAM et al., 2001). 


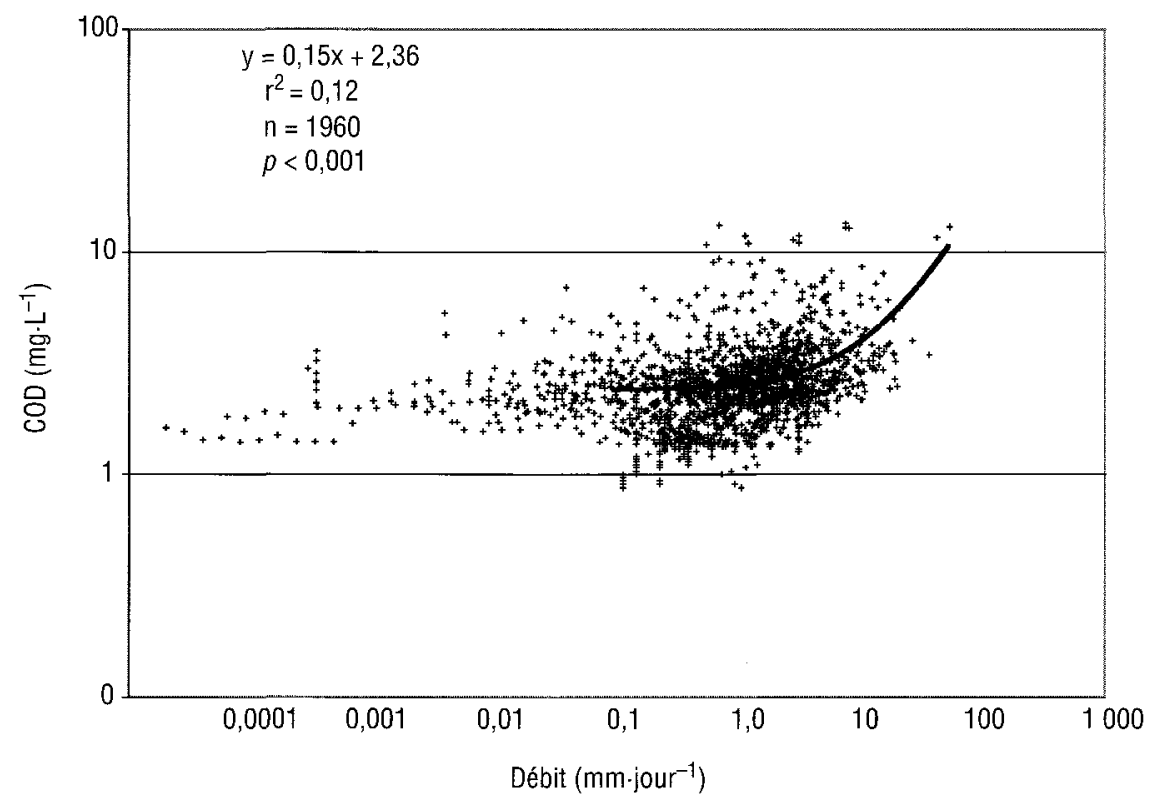

Figure 3 Relation entre le logarithme du débit moyen quotidien (Q) et le logarithme de la concentration de carbone organique dissous (COD) dans le ruisseau de l'Hermine pour les années 1994 à 2003.

Relationship between the logarithm of the mean daily streamflow (Q) and the logarithm of the dissolved organic carbon (DOC) concentrations in the Hermine catchment for the years 1994 to 2003.

En effet, malgré le synchronisme des fluctuations de COD et du débit, l'étendue de la réponse du COD à un changement du débit varie considérablement d'un événement à l'autre. Par exemple, l'événement printanier numéro 2 (figure 2) présente un débit inférieur à $20 \mathrm{~mm} \cdot j o u r-1$ pour un maximum de COD de $4,55 \mathrm{mg} \cdot \mathrm{L}^{-1}$. Par opposition, de faibles fluctuations du débit automnal peuvent engendrer des concentrations maximales annuelles (événement 9; $\mathrm{Q}=2,77 \mathrm{~mm} \cdot \mathrm{jour}^{-1}$ et $\left.\mathrm{COD}=11,23 \mathrm{mg} \cdot \mathrm{L}^{-1}\right)$. Les événements printaniers numéros $12\left(Q=17 \mathrm{~mm} \cdot \mathrm{jour}^{-1}\right.$ et $\left.\mathrm{COD}=3,87 \mathrm{mg} \cdot \mathrm{L}^{-1}\right)$ et $13\left(\mathrm{Q}=32 \mathrm{~mm} \cdot \mathrm{jour}^{-1}\right.$ et $\left.\mathrm{COD}=3,41 \mathrm{mg} \cdot \mathrm{L}^{-1}\right)$, lorsqu'on les compare aux événements automnaux 18 $\left(\mathrm{Q}=5 \mathrm{~mm} \cdot \mathrm{jour}^{-1}\right.$ et $\left.\mathrm{COD}=7,62 \mathrm{mg} \cdot \mathrm{L}^{-1}\right)$ et $19\left(\mathrm{Q}=42 \mathrm{~mm} \cdot \mathrm{jour}^{-1}\right.$ et $\mathrm{COD}=$ $\left.11,46 \mathrm{mg} \cdot \mathrm{L}^{-1}\right)$, illustrent aussi cette variabilité dans la réponse du COD à un changement de débit. En somme, la relation entre les concentrations en COD du ruisseau et le débit varie grandement d'un événement à l'autre et est fonction de l'évolution saisonnière des conditions climatiques et hydrologiques sévissant à l'échelle du bassin de l'Hermine.

\subsection{Saisonnalité de la relation entre le COD et le débit du ruisseau}

Les droites des régressions linéaires ont été tracées pour les 93 événements hydrologiques afin d'illustrer la relation entre la concentration en COD et le débit du ruisseau. La répartition des pentes en deux groupes, en fonction du 
seuil de un, est pratiquement égale (figure 4a). Ainsi, 43 événements ont des pentes COD/Q supérieures à un et 50 événements ont des pentes inférieures à un. L'analyse statistique, sans égard aux saisons, montre que les conditions hydrologiques et climatiques diffèrent significativement entre ces deux groupes (tableau 5). Les événements de pentes supérieures à un se définissent par des conditions hydrologiques sèches et des conditions climatiques plus chaudes et moins pluvieuses. À l'opposé, lors des événements dont la pente est inférieure à un, le bassin présente des conditions plus humides et plus fraîches. À la lumière de ces résultats, une analyse saisonnière a été effectuée afin de mieux dégager les tendances (figures $4 b, c$ et $d$ ).

La majorité des événements de la période hiver-printemps, soit $79 \%$, ont une pente COD/Q nettement inférieure à un (figure $4 \mathrm{~b}$; tableau 6). On notera que l'écart des valeurs de $r^{2}$ de l'unité est principalement lié à l'hystérèse de la relation entre le COD et le débit, tel que montré par BIRON et al. (1999). Cette période est aussi caractérisée par les débits de pointe et moyens les plus élevés, et, donc, par les flux totaux en COD les plus forts (tableau 4) malgré des pics de concentrations en COD excédant à peine $5 \mathrm{mg} \cdot \mathrm{L}^{-1}$. Un groupe de sept épisodes présente cependant des pentes supérieures à un (tableau 6). Tous ont des débits très inférieurs à la moyenne de la saison (figure 4b).

Pour la saison estivale, 20 événements sur 34 ont des pentes supérieures à un (tableau 6). Les concentrations de COD dans le ruisseau sont en moyenne plus élevées et peuvent atteindre des maximums de $12 \mathrm{mg} \cdot \mathrm{L}^{-1}$ (figure $4 \mathrm{c}$ ). Le débit estival moyen représente le débit minimal de l'année (tableau 4). Les variations de débit, même modestes, y sont en général accompagnées d'un changement prononcé de COD (figure 2 : événement 8 ). Certains étés plus humides (données non montrées) présentent des événements où le COD répond plus faiblement à une forte fluctuation du débit. Généralement il s'agit d'événements rapprochés se succédant à l'intérieur d'une période de quelques semaines. Le système étant déjà très humide, la réponse du COD est à ce moment plus faible.

L'automne présente une signature hydrochimique empruntée à la fois à l'été et à la période hiver-printemps (figure 4d). Les maximums de concentration en COD sont aussi élevés et même supérieurs aux maximums estivaux (figure 4c) et les pics de débits sont aussi importants que durant la fonte printanière (figure 4b). Les événements dont la pente COD/Q est supérieure à un (tableau 4) se caractérisent par des débits souvent inférieurs à $5 \mathrm{~mm} \cdot \mathrm{jour}^{-1}$ et des réponses des concentrations de COD élevées (figure 2 : événements 9,10 , 18). Les événements dont la pente COD/Q est faible sont caractérisés par des débits élevés, souvent supérieurs à $10 \mathrm{~mm} \cdot$ jour $^{-1}$ et surviennent lors de pluies automnales successives (figure 2 : événements 19,20 et 21 ). 

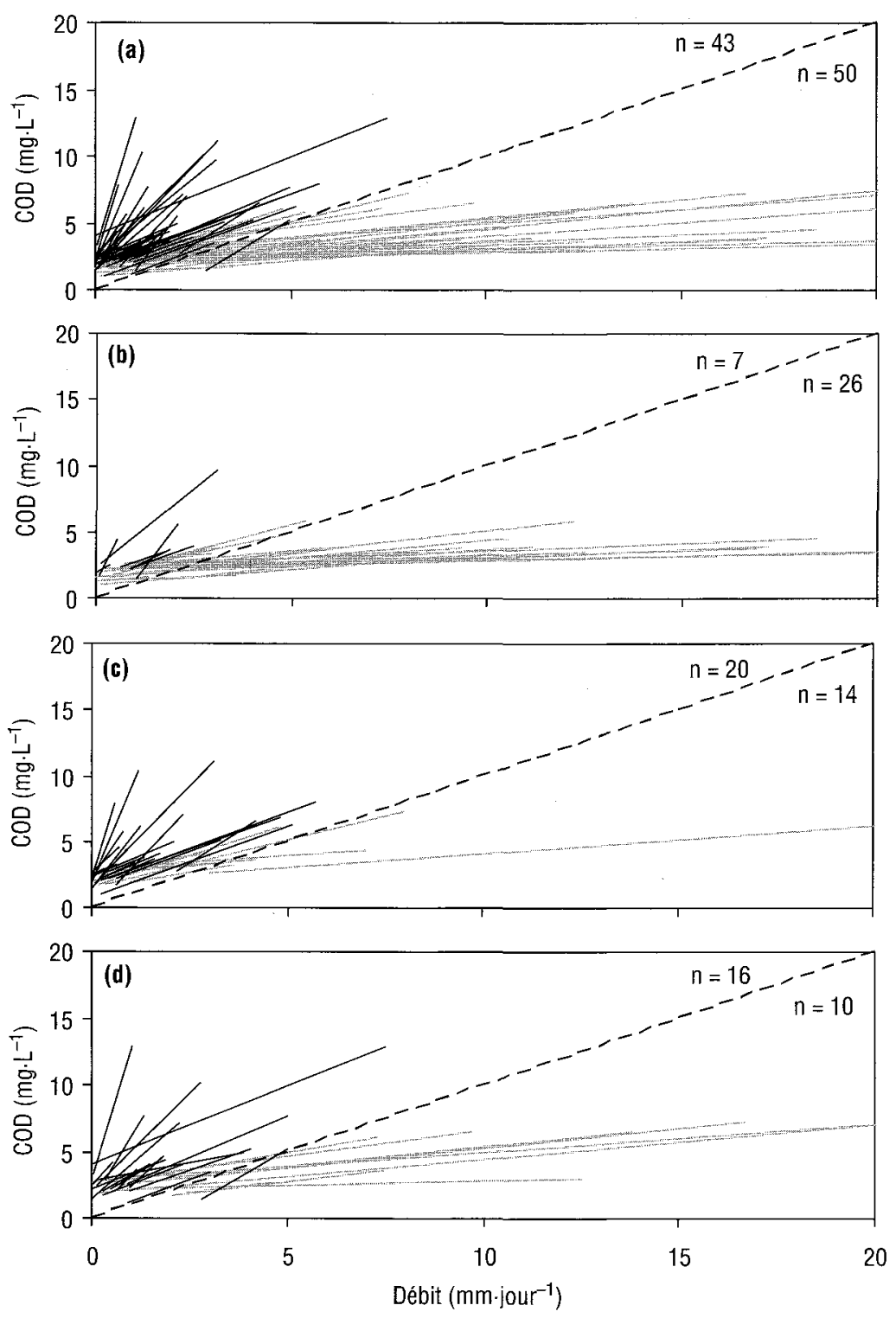

Figure 4 Droites de régression de la relation entre la concentration de carbone organique dissous (COD) et le débit (Q) de l'ensemble des 93 événements hydrologiques (a), pour les événements d'hiver-printemps (b), d'été (c) et d'automne (d) des années 1994 à 2003. Le trait gris représente les événements ayant une pente inférieure à 1 et le trait noir les événements dont les pentes sont supérieures à 1.

Relationship between dissolved organic carbon (DOC) concentration and streamflow (Q) for all the 93 hydrological events (a), winter-spring events (b), summer events (c), and fall events (d) for years 1994 to 2003. The grey lines designate events with slopes lower than 1 and black lines events with slopes higher than 1. 
Tableau 5 Valeurs moyennes (écarts types entre parenthèses) des variables climatiques et hydrologiques influençant les concentrations de carbone organique dissous (COD) et résultats du test de Kruskal-Wallis $(p)$ sur la différence entre les événements de pente COD/Q inférieure à 1 et ceux dont la pente excède 1.

Table 5 Mean values (standard deviations in parentheses) for climatic and hydrological variables influencing the dissolved organic carbon (DOC) concentrations and results of the Kruskal-Wallis test $(\mathrm{p})$ on the annual difference between events with a DOC/Q slope lower than 1 and events with a slope exceeding 1.

\begin{tabular}{|c|c|c|c|c|}
\hline $\begin{array}{l}\text { Variable climatique } \\
\text { et hydrologique }\end{array}$ & $\begin{array}{c}\text { Pente } \\
\text { COD } / \mathrm{Q} \\
\mathrm{mg} \cdot \mathrm{L}^{-1} / \mathrm{mm} \cdot \mathrm{jour} \mathrm{r}^{-1}\end{array}$ & $\stackrel{n}{n}$ événement & $\begin{array}{l}\text { Moyenne } \\
\text { (écart type) }\end{array}$ & $p$ \\
\hline $\begin{array}{l}\text { Précipitation totale durant } \\
\text { événement }\left(\mathrm{m}^{3} \text {. bassin }{ }^{-1}\right)\end{array}$ & $\begin{array}{l}<1,0 \\
>1,0\end{array}$ & $\begin{array}{l}50 \\
43\end{array}$ & $\begin{array}{l}2250(1245) \\
1754(1042)\end{array}$ & 0,029 \\
\hline $\begin{array}{l}\text { Humidité du sol } \\
\text { avant événement (\%) }\end{array}$ & $\begin{array}{l}<1,0 \\
>1,0\end{array}$ & $\begin{array}{l}30 \\
27\end{array}$ & $\begin{array}{l}27,1(3,7) \\
24,4(4,6)\end{array}$ & 0,026 \\
\hline $\begin{array}{l}\text { Profondeur nappe phréatique } \\
\text { avant événement }(\mathrm{cm})\end{array}$ & $\begin{array}{l}<1,0 \\
>1,0\end{array}$ & $\begin{array}{l}46 \\
41\end{array}$ & $\begin{array}{l}80(26) \\
95(23)\end{array}$ & 0,004 \\
\hline $\begin{array}{l}\text { Débit total } 24 \text { h avant événement } \\
\left(\mathrm{m}^{3} \cdot \text { bassin }^{-1}\right)\end{array}$ & $\begin{array}{l}<1,0 \\
>1,0\end{array}$ & $\begin{array}{l}48 \\
41\end{array}$ & $\begin{array}{l}77(85) \\
34(38)\end{array}$ & 0,0003 \\
\hline Température moyenne de l'air $\left({ }^{\circ} \mathrm{C}\right)$ & $\begin{array}{l}<1,0 \\
>1,0\end{array}$ & $\begin{array}{l}50 \\
43\end{array}$ & $\begin{array}{c}7,4(8,7) \\
12,7(6,7)\end{array}$ & 0,003 \\
\hline Température du sol $\left({ }^{\circ} \mathrm{C}\right)$ & $\begin{array}{l}<1,0 \\
>1,0\end{array}$ & $\begin{array}{l}49 \\
43\end{array}$ & $\begin{array}{l}5,8(5,2) \\
9,6(3,6)\end{array}$ & 0,0002 \\
\hline
\end{tabular}

Tableau 6 Pentes et coefficients de détermination $\left(r^{2}\right)$ des relations linéaires obtenues à l'Hermine entre la concentration en carbone organique dissous dans le ruisseau et le débit (COD/Q) pour les 93 événements hydrologiques (1994-2003) groupés sur une base saisonnière.

Table 6 Slope values and determination coefficients $\left(r^{2}\right)$ of the linear relationships obtained at the Hermine catchment between dissolved organic carbon concentrations in the stream and streamflow (DOC/Q) for the 93 hydrological events (1994-2003) grouped on a seasonal basis.

\begin{tabular}{|c|c|c|c|}
\hline Saison & $\begin{array}{c}n \\
\text { événement }\end{array}$ & 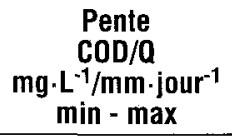 & $\begin{array}{c}\mathrm{r}^{2} \\
\min -\max \end{array}$ \\
\hline $\begin{array}{c}\text { Hiver-Printemps (21 décembre au } 20 \text { juin) } \\
\text { pente }<1,0 \\
\text { pente }>1,0\end{array}$ & $\begin{array}{c}26 \\
7\end{array}$ & $\begin{array}{l}0,06-0,86 \\
1,02-5,76\end{array}$ & $\begin{array}{l}0,26-0,99 \\
0,57-0,99\end{array}$ \\
\hline $\begin{array}{c}\text { Été (21 juin au } 20 \text { septembre) } \\
\text { pente }<1,0 \\
\text { pente }>1,0\end{array}$ & $\begin{array}{l}14 \\
20\end{array}$ & $\begin{array}{l}0,21-0,99 \\
1,05-9,23\end{array}$ & $\begin{array}{l}0,41-0,99 \\
0,30-0,99\end{array}$ \\
\hline $\begin{array}{c}\text { Automne (21 seplembre au } 20 \text { décembre) } \\
\text { pente }<1,0 \\
\text { pente }>1,0\end{array}$ & $\begin{array}{l}10 \\
16 \\
\end{array}$ & $\begin{array}{l}0,07-0,88 \\
1,05-9,74 \\
\end{array}$ & $\begin{array}{l}0,57-0,98 \\
0,64-0,99 \\
\end{array}$ \\
\hline
\end{tabular}




\subsection{Variables climatiques et hydrologiques liées à la relation entre le COD et le débit}

Les liens entre les variables climatiques et hydrologiques, d'une part, et la pente de la relation $C O D / Q$, d'autre part, ont été explorés afin d'identifier les facteurs contrôlant la saisonnalité du transport de COD dans le ruisseau. Le tableau 7 présente les données climatiques et hydrologiques associées aux différences saisonnières entre les événements de pente $C O D / Q$ inférieure à un et ceux dont la pente excède un.

Tableau 7 Valeurs moyennes (écarts types entre parenthèses) des variables climatiques et hydrologiques influençant les concentrations de carbone organique dissous (COD) et résultats du test de Kruskal-Wallis $(p)$ sur la différence saisonnière entre les événements de pente COD/Q inférieure à 1 et ceux dont la pente excède 1 .

Table $7 \quad$ Mean values (standard deviations in parentheses) for climatic and hydrological variables influencing the dissolved organic carbon (DOC) concentrations and results of the Kruskal-Wallis test (p) on the seasonal difference between events with DOC/Q slope lower than 1 and events with slope exceeding 1.

\begin{tabular}{|c|c|c|c|c|c|c|c|c|c|c|}
\hline \multirow[b]{2}{*}{$\begin{array}{l}\text { Variable climatique } \\
\text { et hydrologique }\end{array}$} & \multirow[b]{2}{*}{ Pente } & \multicolumn{3}{|c|}{ Hiver-Printemps } & \multirow[b]{2}{*}{$n$} & \multirow{2}{*}{$\begin{array}{c}\text { Élé } \\
\text { Moyenne } \\
\text { (écart type) }\end{array}$} & \multirow[b]{2}{*}{$p$} & \multirow[b]{2}{*}{$n$} & \multirow{2}{*}{$\begin{array}{c}\text { Automne } \\
\text { Moyenne } \\
\text { (écart type) }\end{array}$} & \multirow[b]{2}{*}{$p$} \\
\hline & & $n$ & $\begin{array}{c}\text { Moyenne } \\
\text { (écart type) }\end{array}$ & $p$ & & & & & & \\
\hline $\begin{array}{l}\text { Précipitation totale } \\
\text { durant événement }\left(\mathrm{m}^{3} \text {. bassin }{ }^{-1}\right.\end{array}$ & $\begin{array}{l}<1,0 \\
>1,0\end{array}$ & $\begin{array}{l}26 \\
7\end{array}$ & $\begin{array}{l}2275(1286) \\
1340(568)\end{array}$ & 0,041 & $\begin{array}{l}14 \\
20\end{array}$ & $\begin{array}{l}1748(1149) \\
1890(1102)\end{array}$ & 0,624 & $\begin{array}{l}10 \\
16\end{array}$ & $\begin{array}{l}2890(1041) \\
1764(1127)\end{array}$ & 0,031 \\
\hline $\begin{array}{l}\text { Humidité du sol } \\
\text { avant événement (\%) }\end{array}$ & $\begin{array}{l}<1,0 \\
>1,0\end{array}$ & $\begin{array}{l}10 \\
2\end{array}$ & $\begin{array}{l}27,4(2,2) \\
32,4(6,6)\end{array}$ & 0,154 & $\begin{array}{l}13 \\
18\end{array}$ & $\begin{array}{l}26,1(4,3) \\
23,1(2,9)\end{array}$ & 0,039 & $\begin{array}{l}7 \\
7\end{array}$ & $\begin{array}{l}24,3(3,4) \\
25,4(6,5)\end{array}$ & 0,749 \\
\hline $\begin{array}{l}\text { Profondeur nappe phréatique } \\
\text { avant événement (cm) }\end{array}$ & $\begin{array}{l}<1,0 \\
>1,0\end{array}$ & $\begin{array}{l}22 \\
7\end{array}$ & $\begin{array}{l}70(22) \\
73(33)\end{array}$ & 1,000 & $\begin{array}{l}14 \\
18\end{array}$ & $\begin{array}{c}96(21) \\
107(12)\end{array}$ & 0,149 & $\begin{array}{l}10 \\
16\end{array}$ & $\begin{array}{l}76(31) \\
92(21)\end{array}$ & 0,190 \\
\hline $\begin{array}{l}\text { Débit total } 24 \mathrm{~h} \text { avant } \\
\text { événement ( } m^{3} \text {.bassin }{ }^{-1} \text { ) }\end{array}$ & $\begin{array}{l}<1,0 \\
>1,0\end{array}$ & $\begin{array}{l}24 \\
7\end{array}$ & $\begin{array}{l}77(92) \\
42(28)\end{array}$ & 0,449 & $\begin{array}{l}14 \\
20\end{array}$ & $\begin{array}{l}87(93) \\
25(37)\end{array}$ & 0,0003 & $\begin{array}{l}10 \\
14\end{array}$ & $\begin{array}{l}63(61) \\
44(42)\end{array}$ & 0,446 \\
\hline $\begin{array}{l}\text { Température moyenne de Yair } \\
\text { (ㄷ) }\end{array}$ & $\begin{array}{l}<1,0 \\
>1,0\end{array}$ & $\begin{array}{c}26 \\
7\end{array}$ & $\begin{array}{l}4,0(8,3) \\
14,6(3,2)\end{array}$ & 0,001 & $\begin{array}{l}14 \\
20\end{array}$ & $\begin{array}{l}16,9(2,0) \\
17,9(2,3)\end{array}$ & 0,146 & $\begin{array}{l}10 \\
16\end{array}$ & $\begin{array}{l}3,0(4,4) \\
5,3(4,6)\end{array}$ & 0,236 \\
\hline $\begin{array}{l}\text { Température du sol } \\
\left({ }^{\circ} \mathrm{C}\right)\end{array}$ & $\begin{array}{l}<1,0 \\
>1,0\end{array}$ & $\begin{array}{c}25 \\
7\end{array}$ & $\begin{array}{l}2,7(3,4) \\
7,7(4,2)\end{array}$ & 0,009 & $\begin{array}{l}14 \\
20\end{array}$ & $\begin{array}{l}12,5(2,7) \\
12,0(1,5)\end{array}$ & 0,958 & $\begin{array}{l}10 \\
16\end{array}$ & $\begin{array}{l}4,6(2,7) \\
7,3(3,3)\end{array}$ & 0,042 \\
\hline
\end{tabular}

Au cours de la période hiver-printemps, le volume de précipitation tombé durant l'événement, la température de l'air et celle du sol sont les variables pour lesquelles les différences entre les deux groupes d'événements sont significatives (tableau 7). En outre, la pente de la relation COD/Q croît avec la température moyenne de l'air et la température du sol (figure 5a). En été, l'humidité du sol et le débit total du cours d'eau 24 h avant l'événement sont les variables qui distinguent les deux groupes (tableau 7). Les changements rapides en COD surviennent lors des événements hydrologiques succédant à une période où des conditions sèches prévalent dans le bassin (figure $5 \mathrm{~b}$ ). Pour les événements d'automne, le volume de précipitation tombé durant l'événement et la température du sol montrent des différences significatives entre les deux groupes (tableau 7). Ainsi, les événements ayant de fortes pentes COD/Q prévalent lorsque la température du sol est plus chaude (figure $5 \mathrm{~d}$ ) et lorsque le volume de précipitation tombé durant l'événement est plus faible (figure $5 \mathrm{c}$ ). 

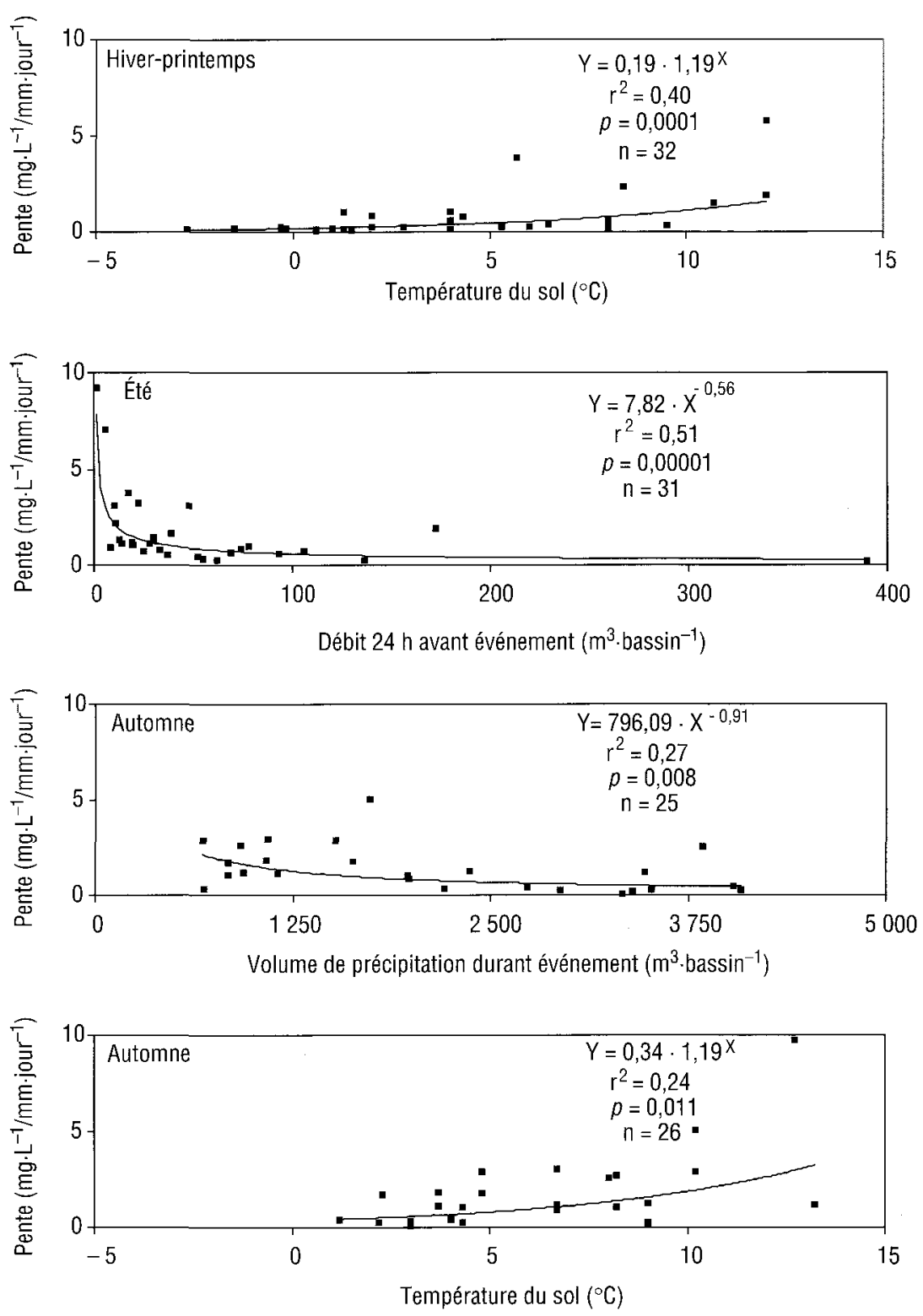

Figure 5 Modélisation saisonnière entre la pente COD/Q et les variables climatique et hydrologiques significatives: (a) température du sol pour la période hiver-printemps, (b) le débit $24 \mathrm{~h}$ avant événement pour la période estivale, (c) le volume de précipitation durant l'événement pour l'automne et (d) la température du sol pour l'automne.

Seasonal modelling for the relationship between DOC/Q and climatic and hydrological variables: (a) mean soil temperature for winter-spring period, (b) streamflow $24 \mathrm{~h}$ before events for summer period, (c) volume of precipitation during event for fall period, and (d) soil temperature for fall period. 


\subsection{Le transport du COD dans le ruisseau de l'Hermine}

L'analyse des relations entre le débit et la concentration de COD dans le ruisseau ainsi que la mise en relief de l'impact différentiel des variables hydrologiques et climatiques sur cette relation suggèrent que les mécanismes qui contrôlent le transport du COD dans le ruisseau de l'Hermine varient à l'échelle saisonnière.

Hiver-printemps. Au cours de la période hiver-printemps, le transport de COD du bassin versant vers le ruisseau est contrôlé par la présence épisodique d'apports élevés en eau à la surface des sols, apports provenant de la fonte de la neige et/ou des pluies (O'BRIEN et HENDERSHOT, 1993). Cette eau contient généralement peu de COD en raison de l'absence de feuillage (pluviolessivats pauvres en COD) et des faibles concentrations en COD de la neige au sol et de la pluie. Lors d'événements hydrologiques majeurs, l'eau d'infiltration pauvre en COD peut toutefois transiter par les horizons de surface riches en matière organique (LFH, Bhf) et s'enrichir en substances organiques solubles qui seront rapidement transportées vers le ruisseau (HENDERSHOT et al., 1996). Ce cheminement hydrologique est responsable de certaines des hausses de COD (jusqu'à $5 \mathrm{mg} \cdot \mathrm{L}^{-1}$ ) observées pendant cette période (figure 2 : événement 2 ). La récurrence de tels événements et la prévalence de températures froides entraînent cependant le lessivage du sol et l'appauvrissement progressif de la réserve en COD soluble. De plus, l'écoulement rapide de l'eau dans les sols lors des crues réduit le temps de contact entre le sol et la solution et ne permet pas à l'eau de s'imprégner de la signature chimique du sol, même si cette eau circule dans les horizons organiques (O'BRIEN et HENDERSHOT, 1993). Ces conditions ont aussi été observées dans d'autres bassins (BOYER et al., 1996) et expliquent la faible réponse en COD mesurée lors de certains épisodes de fonte (figure 2). Pendant l'étiage hivernal, le cours d'eau est alimenté par la nappe phréatique (horizons $\mathrm{BC}$ et $\mathrm{C}$ ) et le niveau de COD se maintient en moyenne en dessous de $2,1 \mathrm{mg} \cdot \mathrm{L}^{-1}$.

Le rôle du volume de précipitation tombé durant l'événement et de la température de l'air et du sol sur les concentrations en COD est manifeste (tableau 7) et a un impact majeur sur le transport du COD dans le ruisseau de l'Hermine au cours de cette saison. En effet, la hausse momentanée des températures de l'air, et par le fait même du sol, favorise la production de COD dans le sol en y augmentant l'activité microbienne et, de là, la décomposition de la matière organique (CHRIST et DAVID, 1996; SOLINGER et al. 2001). La modélisation de la pente $C O D / Q$ versus les variables climatiques et hydrologiques significatives indique que pour la période hiver-printemps la température du sol explique à $40 \%$ le lien existant entre le COD et $Q$ (tableau 8). Ainsi la fonction exponentielle qui en résulte montre qu'une hausse des températures du sol engendre une réponse croissante du COD à un changement de débit (figure 5a). La conjonction d'un redoux et d'un niveau élevé de la nappe phréatique, au moins en bordure du ruisseau, permet alors à l'eau percolant dans les horizons de surface des sols de prendre en charge le COD nouvellement produit, entraînant une hausse prononcée du COD dans le cours d'eau. Les sept événements de cette période présentant une pente COD/Q supérieure à un (figure 4b) illustrent ce mécanisme. II s'agit d'événements tardifs pour la saison (fin mai à mi-juin) marqués par des flux en eau modérés et des températures élevées. 
Tableau 8 Modélisation du logarithme de la pente COD/Q sur une base saisonnière des événements à l'aide des variables climatiques et hydrologiques expliquant le mieux le lien entre le carbone organique dissous (COD) et le débit $(Q)$.

Table 8 Seasonal best-fit from multiple regressions of the logarithm of the $D O C / Q$ against climatic and hydrological variables explaining the link between dissolved organic carbon (DOC) and streamflow (Q).

\begin{tabular}{|c|c|c|c|}
\hline Saison & $\begin{array}{c}\text { Ordonnée } \\
\text { à l'origine } \\
\end{array}$ & Variables & $r^{2}$ \\
\hline Hiver-Printemps & $\begin{aligned}= & -0,73 \pm 0,10^{\mathrm{a}} \\
& (p<0,0001)^{b}\end{aligned}$ & $\begin{array}{c}+0,08 \pm 0,02 \mathbf{t}^{0} \mathbf{s o l}^{\mathrm{c}} \\
(0,0001)\end{array}$ & $\begin{array}{c}n=32 \\
0,40 \\
(0,0001)\end{array}$ \\
\hline Été & $\begin{aligned}= & 0,89 \pm 0,16 \\
& (0,00001)\end{aligned}$ & $\begin{array}{c}-0,56 \pm 0,10 \log _{10} \text { débit } 24 h^{d} \\
(0,00001)\end{array}$ & $\begin{array}{c}n=31 \\
0,51 \\
(0,00001)\end{array}$ \\
\hline Automne & $\begin{array}{c}=2,88 \pm 0,86 \\
(0,003)\end{array}$ & $\begin{array}{c}-1,030 \pm 0,27 \log _{10} \text { vol } \mathbf{P i}^{\mathrm{\theta}}+0,07 \pm 0,02 \mathbf{t}^{0} \text { sol } \\
(0,001) \quad(0,005)\end{array}$ & $\begin{array}{c}n=25 \\
0,50 \\
(0,001)\end{array}$ \\
\hline
\end{tabular}

\footnotetext{
a $: \pm$ valeur $=$ erreur type.

$\mathrm{b}$ : Valeurs entre parenthèses sont les valeurs de $p$ pour chaque paramètre.

${ }^{c}$ : Température du sol exprimé en ${ }^{\circ} \mathrm{C}$.

d : Débit 24 h avant événement exprimé en $\mathrm{m}^{3}$. bassin ${ }^{-1}$.

e : Volume de précipitation durant événement exprimé en $\mathrm{m}^{3} \cdot$ bassin $^{-1}$.
}

Été. Les conditions antécédentes d'humidité régnant dans l'écosystème représentent le principal facteur qui gouverne le transport du COD dans le cours d'eau lors des épisodes hydrologiques d'été. L'effet de la température n'étant pas significatif, la mise en transport du COD dans les sols, plutôt que sa production, semble donc être le facteur contrôlant les concentrations de COD mesurées dans le ruisseau pendant l'été (KALBITZ et al., 2000). Ainsi, lorsque l'humidité du bassin est élevée, comme le soulignent le pourcentage d'humidité des sols et le débit total $24 \mathrm{~h}$ avant l'événement (tableau 7), la pente de la relation $C O D / Q$ est inférieure à un. L'alimentation en eau du ruisseau provient alors des horizons $\mathrm{B}$ et $\mathrm{C}$ dans lesquels la concentration en COD est uniformément faible (tableau 3) en raison de l'effet tampon exercé par le mécanisme d'adsorption anionique du COD (MCDOWELL et LIKENS, 1988). Les variations de COD sont limitées et la concentration moyenne du COD du ruisseau reste sous le niveau de $2,5 \mathrm{mg} \cdot \mathrm{L}^{-1}$. Pendant les événements hydrologiques les plus intenses, le soulèvement soutenu de la nappe peut initier un écoulement dans les horizons organiques et provoquer une hausse du COD dans le ruisseau. La phase de récession et l'étiage qui suivent sont caractérisés par l'accroissement continu de la contribution relative des horizons profonds pauvres en COD au débit du ruisseau. D'autres études ont aussi souligné le lien étroit qui unit les tendances hydrologiques d'écoulement dans les sols, les phases de l'hydrogramme de crue et les variations des concentrations en COD des cours d'eau (ECKHARDT et MOORE, 1990; QUALLS et al., 2002).

Les événements hydrologiques postérieurs à des périodes intenses de sécheresse sont, quant à eux, caractérisés par des pentes COD/Q supérieures à un et par des pics de concentration de COD (tableau 7). Les fortes concen- 
trations de COD mesurées dans le ruisseau ainsi que la rapidité de leur augmentation suggèrent que le COD provient du lessivage de matériaux riches en matière organique soluble localisés à proximité du chenal ou sur son lit. Des propositions similaires ont été soumises par TIPPING et al. (1999). Ces matériaux sources incluent les horizons $B$ podzoliques et les horizons organiques des sols riverains ainsi que les sédiments présents sur le lit du ruisseau. Les solutions extraites des sédiments du ruisseau (données inédites) suggèrent que l'apport du lessivage des sédiments fluviaux à la hausse du COD est probablement mineur dans l'Hermine. D'autres travaux portant sur la contribution des sédiments du lit aux flux en COD montrent des résultats semblables (BUFFAM et al. 2001). En l'absence de sols organiques dans l'Hermine, une fraction significative du COD présent dans le ruisseau lors des crues estivales postérieures aux périodes sèches doit donc être associée à des écoulements de subsurface dans les sols riverains riches en matériaux organiques (MULHOLLAND et HILL, 1997 ; HINTON et al., 1998).

Plusieurs événements estivaux surviennent lorsque le contenu en eau des sols du bassin et le débit antécédent sont bas (tableau 7). Puisque l'écoulement de subsurface, même en marge du chenal, est nul lors des périodes les plus sèches, un autre processus hydrologique doit être envisagé afin d'expliquer les très fortes concentrations en COD mesurées dans le ruisseau (figure 4c). Sous de telles conditions, BIRON et al. (1999) ont proposé que l'hydrophobicité des matériaux organiques des horizons de surface de l'Hermine puisse inhiber temporairement l'infiltration de l'eau dans le sol et favoriser son écoulement rapide à la surface des sols riverains. Cette eau lessive les produits accumulés de la décomposition de la matière organique, s'enrichit fortement en COD et s'écoule vers le chenal. Des événements marqués par de faibles débits et des grandes variations des concentrations en COD surviennent à ce moment. Le comportement hydrophobe des matériaux de surface des sols a été observé dans des bassins versants de milieux secs (BURCH et al., 1989). Des observations directes et fines de ce mécanisme restent à faire pour les milieux forestiers des environnements plus arrosés.

La modélisation de la relation COD/Q pour la période estivale montre que plus de $50 \%$ de la variation est expliqué par le débit $24 \mathrm{~h}$ avant l'événement (tableau 8). La fonction de puissance montre clairement que des débits très faibles (inférieurs à $1 \mathrm{~mm} \cdot \mathrm{jour}^{-1}$ ) génèrent des pics de concentrations en COD parmi les plus élevés de l'année (figure $5 b$ ). Ces observations appuient l'idée de l'existence du phénomène d'hydrophobicité des sols suite à une période prolongée de sécheresse.

Automne. Les données révèlent un comportement hydrochimique parfois similaire à celui de l'été. En effet, plusieurs événements montrent des variations très marquées des concentrations de COD en réponse à une crue et donc, des pentes COD/Q supérieures à un (figure 4d). La température du sol est alors significativement plus élevée que pendant les événements dont la pente est inférieure à un (tableau 7). La production et l'accumulation de substances organiques solubles dans les sols sont donc favorisées et le transport rapide de solutions riches en COD vers le ruisseau s'effectue par le biais d'écoulements dans les horizons supérieurs du B ou dans le LFH, en bordure du ruisseau. La chute récente des feuilles alimente aussi ponctuellement l'eau d'infiltration et celle du ruisseau en COD (HONGVE, 1999; MEYER et al. 1998). 
À l'opposé, d'autres épisodes automnaux ont des pentes COD/Q inférieures à un (figure $4 \mathrm{~d}$ ). Ils surviennent lorsque la température du sol est plus froide et suite à des précipitations nettement plus abondantes que celles reçues lors des événements où le gradient de COD est fort (tableau 7). La parenté avec certains événements de fonte printanière réside dans la faible hausse des concentrations en COD dans le ruisseau lors des crues. Cette réaction s'explique par une production réduite de COD dans les sols sous de basses températures et par le lessivage prononcé des réserves en matières organiques solubles par les eaux de ruissellement pauvres en COD et ce, malgré des écoulements de subsurface abondants. La modélisation (tableau 8) montre que $50 \%$ de la relation COD/Q durant l'automne est expliquée à la fois par le volume de précipitation tombé durant l'événement (figure $5 \mathrm{c}$ ) et par la température du sol (figure $5 \mathrm{~d}$ ).

\subsection{Les changements climatiques et la production et le transport du COD}

La variabilité temporelle du transport du COD est liée à une série de facteurs environnementaux et, en particulier, à la balance entre la productivité primaire de l'écosystème et la décomposition de la matière organique (BROOKS et al., 1999; TRANVICK et JANSSON, 2002). II n'y a actuellement pas de consensus dans la littérature quant à l'ampleur et à la direction de l'impact d'un réchauffement climatique sur la production et le transport du COD. Les perturbations liées à l'activité humaine (coupes forestières, changement d'utilisation du sol, drainage des sols organiques) ajoutent à cette complexité en modifiant la production et les flux de COD dans les écosystèmes (HOUGHTON et al., 1999; GOWER, 2003). Néanmoins, une meilleure compréhension de l'effet des variations saisonnières et événementielles des conditions climatiques et hydrologiques permet de mieux anticiper l'impact d'un changement climatique global sur la production et le transport du COD dans un bassin versant forestier du nord-est du continent nord-américain. Par exemple, nos résultats permettent d'entrevoir, et ce bien que la généralisation à long terme des résultats acquis sur une base événementielle doive se faire avec prudence, qu'un réchauffement climatique peut engendrer une hausse des températures du sol et ainsi favoriser la production de COD dans le sol plus tôt au printemps (MOORE, 1998; FREEMAN et al., 2001). Le réchauffement global peut également augmenter l'évapotranspiration durant l'été et ainsi limiter l'écoulement dans les sols et le ruisseau et, de là, favoriser l'accumulation de produits de décomposition dans les sols, produits qui seront lessivés lors d'événements hydrologiques ultérieurs. Durant la période automnale, l'activité des microorganismes du sol pourra se prolonger si le climat est plus chaud; la production de COD sera alors plus élevée (TRUMBORE et al., 1996). Ces éléments d'interprétation ne signifient pas pour autant que les concentrations de COD dans les écosystèmes aquatiques seront à la hausse. Les changements dans le cheminement de l'eau dans le sol contribuent aussi à établir si le COD sera retenu dans les sols ou s'il sera lessivé vers les écosystèmes aquatiques (MOORE, 1998). En outre, les flux de COD étant fortement liés à l'hydrologie des versants, le transport du COD peut être accentué par des niveaux plus élevés de précipitations (MICHALZIK et al., 2001). 


\section{4 - CONCLUSIONS}

Les résultats montrent que la relation entre le débit du ruisseau de l'Hermine et sa concentration en COD varie fortement sur une base saisonnière. Cette saisonnalité est associée à des variables hydrologiques et climatiques qui conditionnent le transport du COD du bassin versant vers le cours d'eau.

La plupart des événements hydrologiques printaniers présentent des pentes $C O D / Q$ inférieures à un en raison des faibles gradients temporels de COD dans le cours d'eau. Les principales variables contrôlant alors la réponse en COD du bassin sont le volume de précipitation tombé durant les événements, et la température moyenne de l'air et du sol qui régissent la production de COD. La modélisation de la relation COD/Q montre que $40 \%$ de la variation du COD/Q est expliquée par les fluctuations de la température du sol. Lorsque les températures sont élevées pour la saison, le COD est produit en plus grande quantité et l'écoulement de subsurface dans les horizons de sols s'enrichit en COD. Sous des conditions de températures froides, la matière organique soluble est moins abondante et le gradient en COD dans le ruisseau est faible lors des crues. Le lessivage progressif de la réserve en matériaux organiques solubles des sols par les eaux de fonte réduit aussi considérablement les variations en COD du ruisseau.

Pour l'été les conditions d'humidité sévissant dans le bassin avant un épisode hydrologique, comme le pourcentage d'humidité des sols et le débit total $24 \mathrm{~h}$ avant l'événement, constituent les principaux facteurs associés à la magnitude du gradient de COD lors d'une crue. Les changements hydrochimiques les plus prononcés surviennent ainsi suite à une période prolongée de sécheresse. Sous de telles conditions, l'écoulement de subsurface dans les horizons riches en matière organique de sols riverains, l'hydratation des sédiments du lit du ruisseau et le comportement hydrophobe probable des matériaux de surface des sols contribuent, dans des proportions variables, à produire des crues modestes s'accompagnant de très fortes concentrations en COD. Lorsque les conditions antécédentes sont plus humides, l'écoulement provient surtout des horizons $B$ et $C$ et les fluctuations en COD du ruisseau sont très limitées. En appui à ces affirmations, la modélisation obtenue pour l'été indique que $51 \%$ de la variation du COD/Q est expliquée par le débit $24 \mathrm{~h}$ avant l'événement.

À l'automne, le transport de COD dans l'Hermine est influencé par la température du sol, alors plus élevée que celle de l'air, par l'abondance des apports en eau durant les événements et, ponctuellement, par la chute des feuilles. Sur une base événementielle, le comportement hydrochimique automnal du ruisseau peut donc s'apparenter, alternativement, à celui observé en été (suite à des conditions antécédentes sèches) ou à celui rencontré au cours de la saison froide (notamment lors des fortes précipitations). Les régressions multiples indiquent que la fluctuation du COD/Q est expliqué à $50 \%$ par le volume de précipitation durant l'événement et la température du sol.

Les connaissances tirées d'une analyse fine à l'échelle événementielle permettent de mieux comprendre les processus qui gouvernent la production et le transport du COD. L'analyse sous l'angle des variations saisonnières met en 
relief le rôle du climat et de l'hydrologie dans les processus liés et au transport du COD et aide à anticiper l'effet potentiel qu'aura un changement des conditions climatiques globales sur le cycle du carbone dans les écosystèmes forestiers des régions tempérées.

\section{REMERCIEMENTS}

Cette étude a été subventionnée par le Fonds Québécois de Recherche sur la Nature et les Technologies (FQRNT) et le Conseil de Recherches en Sciences Naturelles et en Génie du Canada (CRSNG). Les auteurs aimeraient remercier spécialement Hélène Lalande et Pascale Legrand pour le travail de laboratoire et de terrain ainsi que le personnel de la Station de Biologie de Montréal pour leur appui à nos travaux.

\section{RÉFÉRENCES BIBLIOGRAPHIQUES}

AITKENHEAD J.A., HOPE D., BILLETT M.F., 1999. The relationship between dissolved organic carbon in stream water and soil organic carbon pools at different spatial scales. Hydrol. Process. 13, 1289-1302.

AMUNDSON R., 2001. The carbon budget in soils. Annu. Rev. Earth Planet Sci. 29, 535-562.

ARP P.A., et YIN X., 1992. Predicting water fluxes through forests from monthly precipitation and mean monthly air temperature records. Can. J. For. Res. 22 , 864-877.

BÉLANGER N., CÔTÉ B, COURCHESNE F., FYLES J.W., WARFVINGE P., HENDERSHOT W.H., 2002. Simulation of soil chemistry and nutrient availability in a forested ecosystem of southern Quebec. Part I. Reconstruction of the time-series files of nutrient cycling using the MAKEDEP model. Environmental Modelling and Software. 17, 427-445

BIRON P., ROY A.G., COURCHESNE F., HENDERSHOT W.H., CÔTÉ B., FYLES J., 1999. The effects of antecedent moisture conditions on the relationship of hydrology to hydrochemistry in a small forested watershed. Hydrol. Process. 13, 1541-1555.

BORKEN W., XU Y.J., BRUMME R., LAMERSDORF N., 1999. A climate change scenario for carbon dioxide and dissolved organic carbon fluxes from a temperate forest soil: drought and rewetting effects. Soil Sci. Soc. Am. J. 63, 1848-1855.

BOYER E. W., HORNBERGER G. M., BENCALA K. W., MCKNIGHT D., 1996. Overview of a simple model describing variation of dissolved organic carbon in an upland catchment. Ecol. Modelling. 86, 183-188.

BROOKS P.D., MCKNIGHT D.M., BENCALA K.E. 1999. The relationship between soil heterotrophic activity, soil dissolved organic carbon (DOC) leachate, and catchment-scale DOC export in headwater catchments. Water Resources Research. 35, 1895-1902.

BUFFAM 1., GALLOWAY J. N., BLUM L. K., MCGLATHERY K. J., 2001. A stormflow/baseflow comparison of dissolved organic matter concentrations and bio- 
availability in an Appalachian stream. Biogeochemistry. 53, 269-306.

BURCH G. J., MOORE I. D., BURNS J., 1989. Soil hydrophobic effects on infiltration and catchment runoff. Hydrol. Process. 3, 211-222.

CHRIST M. J., DAVID M. B., 1996. Temperature and moisture effects on the production of dissolved organic carbon in a spodosol. Soil Biol. Biochem. 28, 1191-1199.

Comité d'experts sur la prospection pédologique d'Agriculture Canada, 1987. Le système canadien de classification des sols, $2^{\mathrm{e}}$ édition, Publ. Agriculutre Canada 1646, Ottawa, 164 p.

COURCHESNE F., HENDERSHOT W.H., 1989. Sulfate retention in some podzolic soils of the Laurentians, Quebec. Can. J. Soil Sci. 69, 337-350.

COURCHESNE F., HALLÉ J.P., TURMEL M.C., 2002. Bilans élémentaires holocènes et altération des minéraux dans trois sols forestiers du Québec méridional. Géographie Physique et Quaternaire.s 56, 5-17.

COURCHESNE F., ROY A. G., BIRON P. M, CÔTÉ B., FYLES J., HENDERSHOT W. $H$., 2001. Fluctuations of climatic conditions, elemental cycling and forest growth at the watershed scale. Environ. Monitoring and Assessment. 67, 161177.

CRONAN C. S., AlKEN G. R., 1985. Chemistry and transport of soluble humic substances in forested watersheds of the Adirondack Park, New York. Geochim. Cosmochim. Acta. 49, 1697-1705.

DALVA M., MOORE T. R., 1991. Sources and sinks of dissolved organic carbon in a forested swamp catchment. Biogeochemistry. 15, 1-19.

DOSSKEY M. G., BERTSCH P. M., 1997. Transport of dissolved organic matter through a sandy forest soil. Soil Sci. Soc. Am. J. 61, 920-927.

ECKHARDT B. W., MOORE T. R., 1990. Controls on dissolved organic carbon concentrations in streams southern Quebec. Can. J. Fish. Aquat. Sci. 47, 1537-1544.

FREEMAN G., EVANS D.C., MONTEITH D.T., REYNOLDS B., FENNER N. 2001. Export of organic carbon from peat soils. Nature. $412,785$.
GOWER S.T. 2003. Patterns and mechanisms of the forest carbon cycle. Annual Reviews in Environ. Resources. 28, 169204.

HENDERSHOT W.H., COURCHESNE F., JEFFRIES D.S., 1996. Aluminum geochemistry at the catchment scale in watersheds influenced by acidic precipitation. p. In "The Environmental Chemistry of Aluminum" Sposito, G. [Ed.], CRC Press, Boca Raton, pp. 419-449

HINTON M.J., SCHIFF S.L., ENGLISH M.J., 1998. Sources and flowpaths of dissolved organic carbon during storms in two forested watersheds of the Precambrian Shield. Biogeochemistry. 41, 175-197.

HONGVE D., 1999. Production of dissolved organic carbon in forested catchments. J. Hydrol. 224, 91-99.

HOUGHTON R.A., HACKLER J.L., LAWRENCE K.T. 1999. The U.S. carbon budget: contributions from land-use change. Science. 285, 574-578.

JOBBÁGY E.G., JACKSON R.B., 2000. The vertical distribution of soil organic carbon and its relation to climate and vegetation. Ecol. Appl. 10, 423-432.

KALBITZ K., SOLINGER S., PARK J. H. MICHALZIK B., MATZNER E., 2000. Controls on the dynamics of dissolved organic matter in soils: a review. Soil Sci. 165, 277-304.

KIRSCHBAUM M.U.F., 1995. The temperature dependence of soil organic matter decomposition, and the effect of global warming on soil organic $\mathrm{C}$ storage. Soil Biol. Biochem. 27, 753-760.

KIRSCHBAUM M.U.F., 2000. Will changes in soil organic carbon act as a positive or negative feedback on global warming? Biogeochemistry. 48, 21-51.

MCDOWELL W. H., LIKENS G. E., 1988. Origin, composition, and flux of dissolved organic carbon in the Hubbard Brook Valley. Ecol. Monogr. 58, 177-195.

MCDOWELL W. H., WOOD T., 1984. Podzolization : soil processes control dissolved organic carbon concentrations in stream water. Soil Sci. 137, 23-32.

MCGERRIGLE J. I., 1976. Région de SaintHippolyte/Sainte-Adèle (Québec). Ministère des Richesses Naturelles, Ottawa, Canada, Rapport Géologique $N^{\circ} 179$. $41 \mathrm{p}$. 
MEYER J.L., WALLACE J.B., EGGERT S.L., 1998. Leaf litter as a source of dissolved organic carbon in streams. Ecosystems. 1, 240-249.

MICHALZIK B., KALBITZ K, PARK J.H., SOLINGER S., MATZNER E. 2001. Fluxes and concentrations of dissolved organic carbon and nitrogen - a synthesis for temperate forest. Biogeochemistry. 52, 173-205.

MOORE T. R., 1985. The spectrophotometric determination of dissolved organic carbon in peat waters. Soil Sci. Soc. Am. J. 49, 1590-1592.

MOORE T. R., 1987. An assessment of a simple spectrophotometric method for the determination of dissolved organic carbon in freshwaters. New Zealand Journal of Marine and Freshwater research. 21: 585-589.

MOORE T. R. 1998. Dissolved organic carbon : sources, sinks, and fluxes and role in the soil carbon cycle In Soil Processes and the Carbon Cycle LAL R., KIMBLE J.M., FOLLETT R.F., STEWART B.A. (eds.), Advances in Soil Science, USA.

MULHOLLAND P.J., HILL W.R., 1997. Seasonal patterns in streamwater nutrient and dissolved organic carbon concentrations: separating catchment flow path in stream effects. Water Resour. Res. 33, 1297-1306.

NEFF J.C., ASNER G.P., 2001. Dissolved organic carbon in terrestrial ecosystems : Synthesis and a model. Ecosystems. 4, 29-48.

O'BRIEN C., HENDERSHOT W.H., 1993. Separating streamflow into groundwater, solum and upwelling flow and its implications for hydrochemical modelling. $J$. Hydrol. 146, 1-12.

PRETCHEL A., ALEWELL C., MICHALZIK B., MATZNER E., 2000. Different effect of drying on the fluxes of dissolved organic carbon and nitrogen from a Norway spruce forest floor. J. Plant Nutr. Soil Sci. 163, 517-521.

QUALLS R. G., HAINES B. L., SWANK W. T., TYLER S. W., 2002. Retention of soluble organic nutrients by a forested ecosystem. Biogeochemistry. 61, 135-171.

SAVOIE S., 1988. Contribution of soil solution chemistry to stream water quality in a small forested watershed during spring snowmelt. Master Thesis., McGill University, $103 p$.

SCHIMEL D. S., HOUSE J. I., HIBBARD K. A., BOUSQUET P., CIAIS P., PEYLIN P., BRASWELL B. H., APPS M. J., BAKER D., BONDEAU A., CANADELL J. , CHURKINA G., CRAMER W., DENNING A. S., FIELD C. B., FRIEDLINGSTEIN P. GOODALE C., HEIMANN M., HOUGHTON R. A., MELILLO J. M., MOORE III B., MURDIYARSO D., NOBLE I., PACALA S. W, PRENTICE I. C., RAUPACH M. R., RAYNER P. J., SCHOLES R. J., STEFFEN W. L., WIRTH C., 2001. Recent patterns and mechanisms of carbon exchange by terrestrial ecosystems. Nature. 414, 169-172.

SKOPP J., JAWSON M.D., DORAN J.W., 1990. Steady-state aerobic microbial activity as a function of soil water content. Soil Sci. Soc. Am. J. 54, 16191625.

SOLINGER S., KALBITZ K., MATZNER E., 2001. Controls on the dynamics of dissolved organic carbon and nitrogen in a Central European deciduous forest. Biogeochemistry. 55, 327-349.

SPSS, version 11.5, 2003. Chicago, Illinois, É.U.

STROBEL B.W., BRUUN HANSEN H.C., BORGGAARD O.K., ANDERSEN M.K., RAULUND-RASMUSSEN K., 2001. Composition and reactivity of DOC in forest floor soil solutions in relation to tree species and soil type. Biogeochemistry, 56, 1-26.

TIESSEN H., MOIR J.O., 1993. Total and organic carbon. In "Soil sampling and methods of analysis", Carter, M.R. [Ed.], pp.187-199.

TIPPING E., WOOF C., RIGG E., HARRISSON A. F., INESON P., TAYLOR K., BENHAM D., POSKITT J., ROWLAND A. P., BOL R., HARKNESS D. O., 1999. Climatic influences on the leaching of dissolved organic matter from upland UK moorland soils investigated by a field manipulation experiment. Environ. Int.. 25, 83-95.

TRANVIK L.J., JANSSON M. 2002, Terrestrial export of organic carbon. Nature. 415, 861-862.

TRUMBORE S.E., CHADWICK O.A., AMUNSON R. 1996. Rapid exchange between soil carbon and atmospheric carbon 
dioxide driven by temperature change. Science. 272, 393-396.

VAN CLEVE K., POWERS R.F., 1995. Soil carbon, soil formation and ecosystem development. In "Carbon forms and functions in forest soils" McFee, W.W. et Kelly, J.M. [Ed.], Soil Science Society of America, Madison, pp. 155-200. 